\title{
Anthocyanins and Flavonoids of Vaccinium L.
}

\author{
Zushang Su*
}

National Center for Pharmaceutical Crops, Arthur Temple College of Forestry and Agriculture, Stephen F. Austin State University, Nacogdoches, TX, 75962-6109, USA

\begin{abstract}
Vaccinium L., comprising approximately 450 species primarily in the Northern Hemisphere, is a genus of shrubs or lianas in the family Ericaceae. The berries of many species are harvested for household consumption and commercial sale. The genus produces a wide range of compounds such as anthocyanins, flavonoids, chromones, coumarins, lignans, benzoic acids, iridoids, sterols, and triterpenoids, but is best known for the production of anthocyanins and flavonoids. Extracts and isolates of anthocyanins and flavonoids from Vaccinium fruits or leaves showed antioxidative, anti-inflammatory, antitumor, antiviral, vasoprotective, and antifungal activities. To data, more than 116 anthocyanins and flavonoids compounds have been isolated and identified primarily from the fruits or leaves of Vaccinium. This article reviews phytochemistry and pharmaceutical properties of these compounds.
\end{abstract}

Keywords: anthocyanins, bioactivities, ethnobotany, flavonoids, phytochemistry, Vaccinium L.

\section{INTRODUCTION}

Vaccinium L. (Ericaceae) is a morphologically diverse genus of terrestrial or epiphytic shrubs and lianas, comprising approximately 450 species, which primarily occur in the cooler areas of the northern Hemisphere, although there are tropical species from areas as widely separated as Madagascar and Hawaii [1, 2]. Vaccinium arctostaphylos L. has been used in folk medicine as an antidiabetic and antihypertensive agent [3]. The leaves of rabbiteye blueberry ( $V$. virgatum Aiton, also known as $V$. ashei Reade) have been used in a tea for diabetics among the alpine peasantry. Fruit or leaf extracts of Vaccinium spp. were found to induce apoptosis in cancer cells and to inhibit human leukemia [4-7] and breast [5, 8], colon [4-7, 9, 10], lung [7], and prostate [5, 11, 12] cancer cells in vitro. Vaccinium has been an important source of food and pharmaceutical ingredients coupled with have high antioxidant potential $[10,13,14]$. The berries of many Vaccinium species are harvested for household consumption and commercial sale, particularly of bilberry (V. mytillus L.) $[6,9]$, rabbiteye blueberry [10], lowbush blueberry $(V$. angustifolium Aiton) [11, 12], cranberry (V. macrocarpon Aiton) $[4,5,7]$, and highbush cultivated blueberry $(V$. corymbosum L.) [10, 15]. Today, numerous Vaccinium berry and leaf extract products have been developed as dietary supplements.

The chemical constituents of some of the Vaccinium species have been well documented. The genus produce a wide range of compounds including anthocyanins [3, 16-48], flavonoids [20-23, 28, 37, 40, 42, 46-75], coumarins [73], lignans [76], benzoic acids [77-80], iridoids [81-83], sterols

*Address correspondence to this author at the National Center for Pharmaceutical Crops, Arthur Temple College of Forestry and Agriculture, Stephen F. Austin State University, Nacogdoches, TX 75962, USA;

Tel: 936-468-5646, 936-468-5600; Fax: 936-468-7058;

E-mail: suz@sfasu.edu
[84-87], triterpenoids [52, 55, 84, 88, 89], but are best known for the production of bioactive anthocyanins and flavonoids. To date, more than 116 anthocyanins and flavonoids compounds have been isolated and identified primarily from the fruits and leaves of Vaccinium. This review article focuses on the anthocyanins and flavonoids and their pharmaceutical properties.

\section{ANTHOCYANINS}

Anthocyanins are important plant pigments visible to the human eye. They belong to the widespread class of phenolic compounds collectively named flavonoids. They are glycosides of polyhydroxy and polymethoxy derivatives of 2-phenylbenzopyrylium or flavylium salts [90]. The sugars components of anthocyanins are usually conjugated to the anthocyanin skeleton via the $\mathrm{C}-3$ hydroxyl group in ring $\mathrm{C}$. The differences between individual anthocyanins relate to the number of hydroxyl groups, the nature and the number of sugars attached to the molecule, the position of this attachment, and the nature and the number of aliphatic or aromatic acids attached to the sugars in the molecule [91].

The genus Vaccinium has been shown to contain high levels and a wide variety of anthocyanins that provide the red, blue, purple, and black colors of these berries [92]. Many studies have been examed the contents and the composition of anthocyanins of Vaccinium species in the last two decades. The isolation and the structural elucidation of the individual ingredients in the anthocyanin mixtures from the extract of this genus have been the target of many investigations. The isolated anthocyanins are highly unstable and very susceptible to degradation. Their stability is affected by several factors such as $\mathrm{PH}$, storage temperature, chemical structure, concentration, light, oxygen, solvents, and the presence of enzymes, proteins and metallic ions. The characterization of a mixture of anthocyanins usually involves the separation and collection of each compound, and subsequent analysis by nuclear magnetic resonance and 


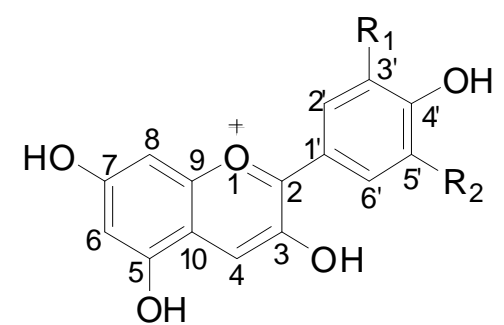

\begin{tabular}{|c|c|c|}
\hline Name & $\mathbf{R}_{\mathbf{1}}$ & $\mathbf{R}_{\mathbf{2}}$ \\
\hline Delphinidin & $\mathrm{OH}$ & $\mathrm{OH}$ \\
\hline Petunidin & $\mathrm{OCH}_{3}$ & $\mathrm{H}$ \\
\hline Cyanidin & $\mathrm{OH}$ & $\mathrm{H}$ \\
\hline Pelargonidin & $\mathrm{H}$ & $\mathrm{H}$ \\
\hline Peonidin & $\mathrm{OCH}_{3}$ & $\mathrm{H}$ \\
\hline Malvidin & $\mathrm{OCH}_{3}$ & $\mathrm{OCH}_{3}$ \\
\hline
\end{tabular}

Fig. (1). Chemical structure of anthocyanidins.

fast atom bombardment mass spectroscopy. For the separation and structural analysis, the use of liquid chromatography-mass spectrometry technique, which combines the separation of LC with the selectively and sensitivity of the MS detector, permits the identification of individual compounds in the mixture of compounds [93]. The most common anthocynidins are delphinidin, cyaniding, peonidin, petunidin and malvidin, all of them being found in Vaccinium berries. Galactose, glucose, arabinose, xylose, and rhamnose are the most common sugars that are bonded to anthocyanidins in mono-, di-, or trisaccharide forms. To date, a total of 41 naturally occurring anthocyanidins or aglycones were reported in the literature. In this review, the name, source and references are listed in Table $\mathbf{1 .}$

Six anthocyanidins (1-6), pelarogonidin, cyanidin, petunidin, delphinidin, peonidin, and malvidin were isolated from the genus Vaccinium, which are also the most common anthocyanidin skeletons in higher plants (Fig. 1).

The glycoside derivatives of the six anthocyanidins are the most common in nature. The following four classes of anthocyanidins glycosides are common: 3-monoside, 3biosides, 3,5-diglycosides and 3,7-diglycosides. To date, about 35 anthocyanin glycosides have been isolated and identified from the genus Vaccinium (7-41). The anthocyanin glycosides include two pelargonidin glycosides, nine cyanidin glycosides, six peonidin glycosides, six delphinidin glycosides, six petunidin glycosides, and six malvidin glycosides. Most of the anthocyanins have a monosaccharide unit attached to the C-3 position of the aglycone. Some of them have a disaccharide or trisaccharide chain at C-3 of the anthocyanins. It is well-known that the most common nature of the sugar is glucose, galactose, arabinose, rhamnose, and xylopyranose.

In the past only the 3-O-arabinoside (7) and 3-O-xyloside (8) of pelargonidin have been isolated from the berries of $V$. japonicum [32]. Cyanidin and its glycosides are present in both subgenus Oxycoccus (cranberries) and various sections of subgenus Vaccinium. Compounds cyaniding-5-Oglucoside (13) and cyaniding-3,5-O-diglucoside (14) have been reported from $V$. myrtillus containing a 5 -glucoside and 3,5-diglucosides respectively. Eight disaccharides cyanidin 3-O-gentiobioside (15), 3-O-sambutioside of cyanidin (16), peonidin (22), delphinidin (28), petunidin (34) and malvidin (40), petunidin 3-O-rutinoside (35) and malvidin (41), and three trisaccharides cyanidin 3-O-(6"-O-2-rhamnopyranpsyl$2 "-O$ - $\beta$-xylopranosyl- $\beta$-glucopyranoside) (17), peonidin (23), and delphinidin (29) have been found previously in the genus Vaccinium [24]. It is somewhat ironic that the blueberry contains much cyanidin compound, as cyanidin is usually associated with red flowers [94]. Recently, Ballinger et al. reported the extraction and purification of anthocyanins from $V$. arboretum. The compounds were identified as the 3monoglycosides of the aglycons delphinidin, petunidin, malvidin, cyanidin, and peonidin with the sugars arabinose, galactose, and glucose (except for cyanidin and malvidin). $V$. arboreum fruit contains anthocyanins which are extremely similar to those reported for the fruits of highbush and lowbush blueberries. $V$. arboreum has at least 12 anthocyanins, while those of $V$. stamineum whose geog. range is similar to that of $V$. arboreum, has only 3 (monoglycosides of the sugars galactose, arabinose, or glucose with the aglycon cyanidin) [19]. Twenty anthocyanins were isolated from the extract of the edible blue berries of $V$. padifolium (Uveira) by a combination of chromatography techniques. They were identified as the 3$O$ - $\beta$-glucopyranosides, 3-O- $\beta$-galactopyranosides, $3-O-\beta$ arabinopyranosides, and $3-O-\beta$-sambubiosides (2"- $O-\beta$ xylopyranosyl- $O-\beta$-glucopyranosides) of delphinidin, cyanidin, petunidin, malvidin, and peonidin. Among them, 3$O$-sambubiosides of peonidin, petunidin and malvidin are first reported as anthocyanidin disaccharides from the genus Vaccinium. However, petunidin (5) and Malvidin (6), and their glycosides (30-35 and 36-41, respectively) are reported in subgenus Vaccinium only, some non glucoside and galactoside may restrict to specific species [24].

Table 1. The Names, Sources, and References of Compounds 1-41

\begin{tabular}{|c|c|c|c|}
\hline No. & Name & Source & References \\
\hline \hline $\mathbf{1}$ & Pelargonidin & V. japonicum (fruit) & {$[32]$} \\
\hline $\mathbf{2}$ & Cyanidin & V. $\times$ intermedium (fruit) & {$[48]$} \\
\hline & & V. myrtillus (fruit) & {$[21,43,45,46]$} \\
\hline & & V. corymbosum (fruit) & {$[46]$} \\
\hline $\mathbf{3}$ & Peonidin & V. padifolium (fruit) & {$[24]$} \\
\hline $\mathbf{4}$ & Delphinidin & V. myrtillus (leaves \& fruit) & {$[46]$} \\
\hline $\mathbf{5}$ & Petunidin & V. $\times$ intermedium (fruit) & {$[48]$} \\
\hline
\end{tabular}


Table 1. cont...

\begin{tabular}{|c|c|c|c|}
\hline No. & Name & Source & References \\
\hline 6 & Malvidin & V. padifolium (fruit) & [24] \\
\hline 7 & Pelargonidin-3-O-arabinoside & V. japonicum (fruit) & {$[32]$} \\
\hline 8 & Pelargonidin-3-O-xyloside & V. japonicum (fruit) & {$[32]$} \\
\hline \multirow[t]{15}{*}{9} & Cyanidin-3-O-arabinoside & V. macrocarpon (fruit) & {$[69,77]$} \\
\hline & & V. angustifolium (fruit) & [29] \\
\hline & & V. virgatum $=V$. ashei $($ fruit $)$ & {$[20]$} \\
\hline & & V. corymbosum (fruit) & {$[18,46,95]$} \\
\hline & & V. $\times$ covilleanum (fruit) & [37] \\
\hline & & V. myrtillus (fruit) & {$[21,43,45,46]$} \\
\hline & & V. membranaceum (fruit) & {$[96]$} \\
\hline & & V. padifolium (fruit) & {$[24]$} \\
\hline & & V. ovatum (fruit) & [96] \\
\hline & & V. uliginosum (fruit) & [33] \\
\hline & & V. vitis-idaea (fruit) & {$[31,38,42]$} \\
\hline & & V. $\times$ intermedium (fruit) & {$[48]$} \\
\hline & & V. meridionale (fruit) & [47] \\
\hline & & V. oxycoccus $L$. (fruit) & {$[36]$} \\
\hline & & V. stamineum L. (fruit) & {$[30]$} \\
\hline \multirow[t]{14}{*}{10} & Cyanidin-3-O-galactoside & V. macrocarpon (fruit) & {$[69,77]$} \\
\hline & & V. angustifolium (fruit) & [29] \\
\hline & & V. virgatum $=V$. ashei $($ fruit $)$ & {$[20]$} \\
\hline & & V. corymbosum (fruit) & {$[18,46,95]$} \\
\hline & & V. $\times$ covilleanum (fruit) & [37] \\
\hline & & V. myrtillus (fruit) & {$[17,21]$} \\
\hline & & V. membranaceum (fruit) & [96] \\
\hline & & V. padifolium (fruit) & [24] \\
\hline & & V. ovatum (fruit) & [96] \\
\hline & & V. uliginosum (fruit) & [33] \\
\hline & & V. vitis-idaea (fruit) & {$[31,39]$} \\
\hline & & V. $\times$ intermedium (fruit) & {$[22,48]$} \\
\hline & & V. oxycoccus $L$. (fruit) & {$[36]$} \\
\hline & & V. stamineum L. (fruit) & [30] \\
\hline \multirow[t]{3}{*}{11} & Cyanidin-3-O-xyloside & V. arctostaphylos (fruit) & [97] \\
\hline & & V. angustifolium (fruit) & [29] \\
\hline & & V. myrtillus (fruit) & {$[38,43,45,46]$} \\
\hline \multirow[t]{12}{*}{12} & Cyanidin-3-O-glucoside & V. macrocarpon (fruit) & {$[69,77]$} \\
\hline & & V. angustifolium (fruit) & [29] \\
\hline & & V. virgatum $=V$. ashei $($ fruit $)$ & {$[20,41]$} \\
\hline & & V. corymbosum (fruit) & {$[16,37,95]$} \\
\hline & & V. $\times$ covilleanum (fruit) & [37] \\
\hline & & V. myrtillus (fruit) & {$[17,21]$} \\
\hline & & V. membranaceum (fruit) & [96] \\
\hline & & V. padifolium (fruit) & [24] \\
\hline & & V. ovatum (fruit) & [96] \\
\hline & & V. uliginosum (fruit) & {$[33,40]$} \\
\hline & & V. vitis-idaea (fruit) & [31] \\
\hline & & V. $\times$ intermedium (fruit) & {$[22,48]$} \\
\hline
\end{tabular}


Table 1. cont...

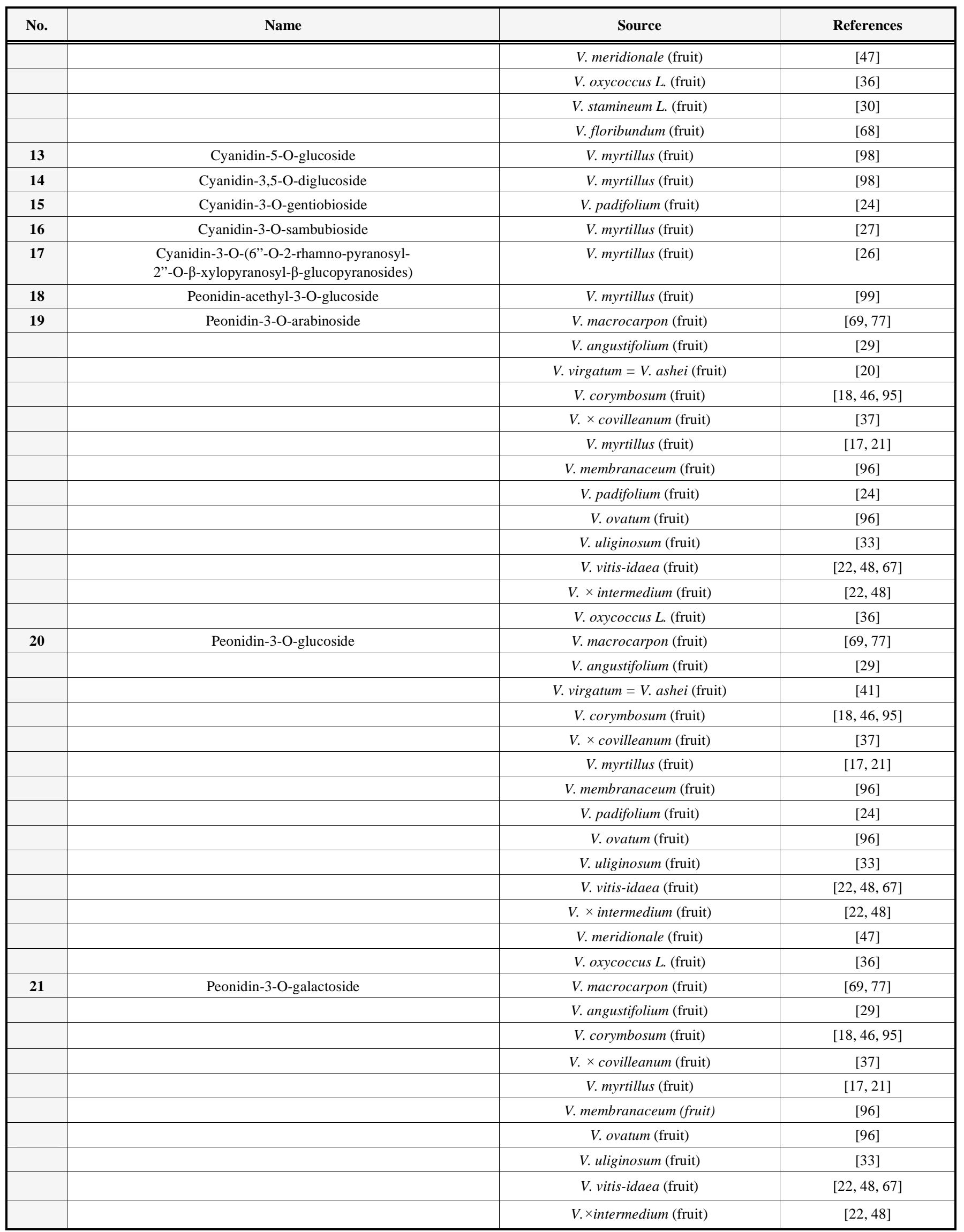


Table 1. cont...

\begin{tabular}{|c|c|c|c|}
\hline No. & Name & Source & References \\
\hline & & V. oxycoccus $L$. (fruit) & [36] \\
\hline 22 & Peonidin-3-O-sambubioside & V. padifolium (fruit) & [24] \\
\hline 23 & $\begin{array}{c}\text { Peonidin-3-O-(6"-O-2-rhamnopyranosyl-2"- } \\
\text { O- } \beta \text {-xylopyranosyl- } \beta \text {-glucopyranosides) }\end{array}$ & V. padifolium (fruit) & [26] \\
\hline \multirow[t]{12}{*}{24} & Delphinidin-3-O-arabinoside & V. macrocarpon (fruit) & {$[69,77]$} \\
\hline & & V. angustifolium (fruit) & [29] \\
\hline & & $V$. virgatum $=V$. ashei $($ fruit $)$ & [20] \\
\hline & & V. corymbosum (fruit) & {$[18,46,95]$} \\
\hline & & V. $\times$ covilleanum (fruit) & [37] \\
\hline & & V. myrtillus (fruit) & {$[17,21]$} \\
\hline & & V. membranaceum (fruit) & [96] \\
\hline & & V. ovatum (fruit) & [96] \\
\hline & & V. uliginosum (fruit) & [33] \\
\hline & & V. vitis-idaea (fruit) & {$[22,48,67]$} \\
\hline & & $V . \times$ intermedium (fruit) & {$[22,48]$} \\
\hline & & V. padifolium (fruit) & [24] \\
\hline 25 & Delphinidin-3-O-xyloside & V. arctostaphylos (fruit) & [97] \\
\hline \multirow[t]{12}{*}{26} & Delphinidin-3-O-galactoside & V. macrocarpon (fruit) & {$[69,77]$} \\
\hline & & V. angustifolium (fruit) & [29] \\
\hline & & $V$. virgatum $=V$. ashei $($ fruit $)$ & [20] \\
\hline & & V. corymbosum (fruit) & {$[18,46,95]$} \\
\hline & & V. $\times$ covilleanum (fruit) & [37] \\
\hline & & V. myrtillus (fruit) & {$[17,21]$} \\
\hline & & V. membranaceum (fruit) & [96] \\
\hline & & V. ovatum (fruit) & [96] \\
\hline & & V. uliginosum (fruit) & [33] \\
\hline & & V. vitis-idaea (fruit) & {$[22,48,67]$} \\
\hline & & $V . \times$ intermedium (fruit) & {$[22,48]$} \\
\hline & & V. padifolium (fruit) & [24] \\
\hline \multirow[t]{15}{*}{27} & Delphinidin-3-O-glucoside & V. macrocarpon (fruit) & {$[69,77]$} \\
\hline & & V. angustifolium (fruit) & [29] \\
\hline & & $V$. virgatum $=V$. ashei (fruit) & [20] \\
\hline & & V. corymbosum (fruit) & {$[18,46,95]$} \\
\hline & & V. $\times$ covilleanum (fruit) & [37] \\
\hline & & V. myrtillus (fruit) & {$[17,21]$} \\
\hline & & V. membranaceum (fruit) & [96] \\
\hline & & V. arctostaphylos (fruit) & [97] \\
\hline & & V. padifolium (fruit) & [24] \\
\hline & & V. ovatum (fruit) & [96] \\
\hline & & V. uliginosum (fruit) & {$[33,100]$} \\
\hline & & V. vitis-idaea (fruit) & {$[31]$} \\
\hline & & V. $\times$ intermedium (fruit) & {$[22,48]$} \\
\hline & & V. oxycoccus $L$. (fruit) & [36] \\
\hline & & V. floribundum (fruit) & [68] \\
\hline 28 & Delphinidin-3-O-sambubioside & V. myrtillus (fruit) & [27] \\
\hline 29 & $\begin{array}{l}\text { Delphinidin-3-O-(6"-O-2-rhamnopy ranosyl- } \\
2 \text { "-O- } \beta \text {-xylopyranosyl- } \beta \text {-glucopyranosides) }\end{array}$ & V. padifolium (fruit) & [26] \\
\hline 30 & Petunidin-3-O-arabinoside & V. myrtillus (fruit) & [21] \\
\hline
\end{tabular}


Table 1. cont...

\begin{tabular}{|c|c|c|c|}
\hline No. & Name & Source & References \\
\hline & & V. corymbosum (fruit) & {$[18,46,95]$} \\
\hline & & V. uliginosum (fruit) & {$[29,33]$} \\
\hline & & $V$. virgatum $=V$. ashei (fruit) & [20] \\
\hline \multirow[t]{2}{*}{31} & Petunidin-3-O-xyloside & V. arctostaphylos (fruit) & {$[101]$} \\
\hline & & V. $\times$ intermedium (fruit) & {$[48]$} \\
\hline \multirow[t]{9}{*}{32} & Petunidin-3-O-galactoside & V. angustifolium (fruit) & [29] \\
\hline & & V. corymbosum (fruit) & {$[18,46,95]$} \\
\hline & & V. myrtillus (fruit) & {$[17,21]$} \\
\hline & & V. arctostaphylos (fruit) & [97] \\
\hline & & V. padifolium (fruit) & {$[24]$} \\
\hline & & V. uliginosum (fruit) & {$[100]$} \\
\hline & & V. vitis-idaea (fruit) & {$[22,48,67]$} \\
\hline & & V. $\times$ intermedium (fruit) & {$[22,48]$} \\
\hline & & V. virgatum $=V$. ashei (fruit) & {$[20]$} \\
\hline \multirow[t]{10}{*}{33} & Petunidin-3-O-glucoside & V. angustifolium (fruit) & [29] \\
\hline & & V. corymbosum (fruit) & {$[18,19,46,95]$} \\
\hline & & V. myrtillus (fruit) & {$[17,21]$} \\
\hline & & V. arctostaphylos (fruit) & {$[97]$} \\
\hline & & V. padifolium (fruit) & {$[24]$} \\
\hline & & V. uliginosum (fruit) & {$[33,100]$} \\
\hline & & V. vitis-idaea (fruit) & {$[22,48,67]$} \\
\hline & & V. $\times$ intermedium (fruit) & {$[22,48]$} \\
\hline & & V. oxycoccus $L$. & [36] \\
\hline & & $V$. virgatum $=V$. ashei (fruit) & {$[20]$} \\
\hline 34 & Petunidin-3-O-sambubioside & V. padifolium (fruit) & {$[24]$} \\
\hline 35 & Petunidin-3-O-rutinoside & V. padifolium (fruit) & {$[24]$} \\
\hline \multirow[t]{3}{*}{36} & Malvidin-3-O-arabinoside & V. myrtillus (fruit) & {$[21]$} \\
\hline & & V. corymbosum (fruit) & {$[18,19,46,95]$} \\
\hline & & V. uliginosum (fruit) & {$[33,100]$} \\
\hline 37 & Malvidin-3-O-xyloside & V. arctostaphylos (fruit) & {$[101]$} \\
\hline \multirow[t]{8}{*}{38} & Malvidin-3-O-galactoside & V. angustifolium (fruit) & {$[29]$} \\
\hline & & V. corymbosum (fruit) & {$[18,19,46,95]$} \\
\hline & & V. myrtillus (fruit) & {$[17,21]$} \\
\hline & & V. arctostaphylos (fruit) & [97] \\
\hline & & V. uliginosum (fruit) & {$[40,100]$} \\
\hline & & V. vitis-idaea (fruit) & {$[22,48,67]$} \\
\hline & & $V . \times$ intermedium (fruit) & {$[22,48]$} \\
\hline & & V. virgatum $=V$. ashei (fruit) & {$[20]$} \\
\hline \multirow[t]{9}{*}{39} & Malvidin-3-O-glucoside & V. angustifolium (fruit) & {$[29]$} \\
\hline & & V. corymbosum (fruit) & {$[37,95]$} \\
\hline & & V. myrtillus (fruit) & {$[17,21]$} \\
\hline & & V. arctostaphylos (fruit) & {$[97]$} \\
\hline & & V. uliginosum (fruit) & {$[33,40,100]$} \\
\hline & & V. vitis-idaea (fruit) & {$[22,48,67]$} \\
\hline & & $V . \times$ intermedium (fruit) & {$[22,48]$} \\
\hline & & $V$. virgatum $=V$. ashei (fruit) & {$[20]$} \\
\hline & & V. oxycoccus L. (fruit) & [36] \\
\hline 40 & Malvidin-3-O-sambubioside & V. padifolium (fruit) & {$[24]$} \\
\hline 41 & Malvidin-3-O-rutinoside & V. padifolium (fruit) & {$[24]$} \\
\hline
\end{tabular}


<smiles>Oc1ccc(-c2[o+]c3cc(O)cc(O)c3cc2O)cc1</smiles>

1<smiles></smiles>

4<smiles></smiles>

7<smiles>Oc1cc(O)c2cc(OC3OC[C@@H](O)[C@H](O)[C@H]3O)c(-c3ccc(O)c(O)c3)[o+]c2c1</smiles><smiles>Oc1cc(O)c2cc(O)c(-c3ccc(O)c(O)c3)[o+]c2c1</smiles>

2<smiles></smiles>

5<smiles>OC[C@H]1OC(Oc2cc3c(O)cc(O)cc3[o+]c2-c2ccc(O)cc2)[C@H](O)[C@@H](O)[C@H]1O</smiles>

8<smiles>OC[C@H]1O[C@@H](Oc2cc3c(O)cc(O)cc3[o+]c2-c2ccc(O)c(O)c2)[C@H](O)[C@@H](O)[C@H]1O</smiles><smiles>COc1cc(-c2[o+]c3cc(O)cc(O)c3cc2O)ccc1O</smiles>

3<smiles></smiles>

6<smiles>Oc1cc(O)c2cc(OC3OC[C@@H](O)[C@H](O)[C@H]3O)c(-c3ccc(O)c(O)c3)[o+]c2c1</smiles>

9<smiles>OC[C@H]1O[C@@H](Oc2cc3c(O)cc(O)cc3[o+]c2-c2ccc(O)c(O)c2)[C@H](O)[C@@H](O)[C@H]1O</smiles>

10<smiles>OC[C@H]1OC(Oc2cc(O)cc3[o+]c(-c4ccc(O)c(O)c4)c(O)cc23)C(O)[C@@H](O)[C@@H]1O</smiles>

13<smiles>OC[C@H]1OC(Oc2cc(O)cc3[o+]c(-c4ccc(O)c(O)c4)c(OC4O[C@H](CO)[C@@H](O)[C@H](O)C4O)cc23)[C@H](O)[C@@H](O)C1O</smiles> 
<smiles>OC[C@H]1O[C@H](OC[C@H]2O[C@@H](Oc3cc4c(O)cc(O)cc4[o+]c3-c3ccc(O)c(O)c3)[C@H](O)[C@@H](O)[C@@H]2O)[C@H](O)[C@@H](O)[C@@H]1O</smiles>

15

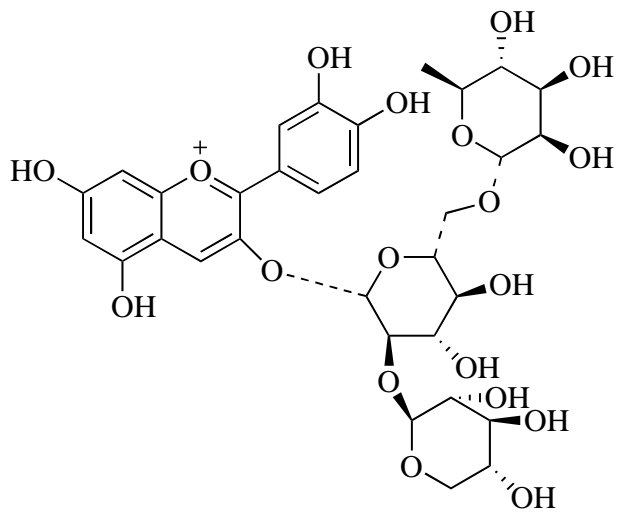

17<smiles>COc1cc(-c2[o+]c3cc(O)cc(O)c3cc2O[C@@H]2OC[C@@H](O)[C@H](O)[C@H]2O)ccc1O</smiles>

19<smiles></smiles>

22<smiles>OC[C@H]1OC(Oc2cc3c(O)cc(O)cc3[o+]c2-c2ccc(O)c(O)c2)[C@H](O[C@H]2C[C@H](O)[C@@H](O)CO2)[C@H](O)[C@@H]1O</smiles>

16<smiles></smiles>

18<smiles>COc1cc(C2=C(OC3O[C@H](CO)[C@@H](O)[C@H](O)[C@H]3O)C=C3[C](C=C(O)C=C3O)O2)ccc1O</smiles>

21<smiles></smiles>

23 
<smiles>Oc1cc(O)c2cc(OC3OC[C@@H](O)[C@H](O)[C@H]3O)c(-c3cc(O)c(O)c(O)c3)[o+]c2c1</smiles>

24<smiles>Oc1cc(O)c2cc(O[C@@H]3OC[C@@H](O)[C@H](O)[C@H]3O)c(-c3cc(O)c(O)c(O)c3)[o+]c2c1</smiles>

25<smiles>O=C(/C(=C\c1c(O)cc(O)cc1[OH+])OC1O[C@H](CO)[C@@H](O)[C@H](O)[C@H]1O)c1cc(O)c(O)c(O)c1</smiles>

26<smiles>OC[C@H]1O[C@@H](Oc2cc3c(O)cc(O)cc3[o+]c2-c2cc(O)c(O)c(O)c2)[C@H](O)[C@@H](O)[C@@H]1O</smiles>

27<smiles></smiles>

30<smiles>COc1cc(C(=O)/C(=C/c2c([O-])cc(O)cc2O)O[C@@H]2O[C@H](CO)[C@@H](O)[C@H](O)[C@H]2O)cc(O)c1O</smiles><smiles></smiles><smiles></smiles>

31 32<smiles></smiles> 
<smiles>COc1cc(C(=O)c2cc3c(O)cc(O)cc3[o+]c2OC2OC[C@@H](O)[C@H](O)[C@H]2O)cc(OC)c1O</smiles>

36<smiles></smiles>

37<smiles></smiles>

38<smiles></smiles>

39<smiles></smiles>

40<smiles>COc1cc(C(=O)c2cc3c(O)cc(O)cc3[o+]c2OC2O[C@H](COC3O[C@H](C)[C@@H](O)[C@H](O)[C@H]3O)[C@@H](O)[C@H](O)[C@H]2O)cc(OC)c1O</smiles>

41

\section{FLAVONOIDS}

Flavonoids are formed in plants from the aromatic amino acids phenylalanine and tyrosine, and malonate. The basic flavonoid structure is the flavan nucleus, which consists of 15 carbon atoms arranged in three rings $\left(\mathrm{C}_{6}-\mathrm{C}_{3}-\mathrm{C}_{6}\right)$. Which are labeled A, B, and C (Fig. 2). The various classes of flavonoids differ in the level of oxidation and pattern of substitution of the $\mathrm{C}$ ring, while individual compounds within a class differ in the pattern of substitution of the A and $\mathrm{B}$ rings. Flavonoids are among the most ubiquitous phenolic compounds found in the genus Vaccinium. Their structures vary based on the substituents of hydroxyl at B ring, as well as the nature and the number of sugars attached to the molecule. To data, more than 50 compounds have been isolated and identified from the genus Vaccinium. Flavonoid glycosides are one of the most frequently chemical constituents from Vaccinium. The most common

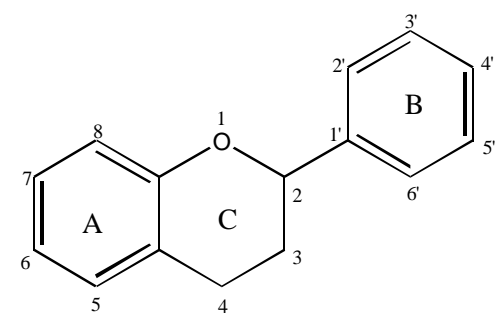

Fig. (2). Basic flavonoid structure. sugar moieties include D-glucose, L-ahamnose, D-xylose, Dgalactose, and L-arabinose. The glycosides are usually $O$ glycosides, with the sugar moiety bound to the hydroxyl group at C-3 or C-7 position. The most common flavonol in the Vaccinium is quercetin.

\section{Flavone, Flavonol and their Glycosides}

Flavone, flavonol and their glycosides are the main chemical constituents from genius Vaccinium. The glycosides are usually $O$-glycosides, with the sugar moiety bound to the hydroxyl group at the C-3 or C-7 position. A total of 45 flavonoids including flavone, flavonol and their glycosides were isolated and identified from the genus Vaccinium (Table 2).

A few early investigations from the leaves of $V$. myrtillus led to the isolation and identification of quercetin, quercetin 3-arabinoside, isoquercitrin, quercetin 3-rhamnoside, quercetin 3-glucoglucoside, and apparently a new quercetin arabinoside not identical with quercetin 3-arabinoside [21, 50]. It is interesting that four novel flavonols with a 3-Oglucuronic acid were isolated from the species $V$. bracteatum, $V . \times$ intermedium and $V$. myrtillus [22]. During the past decade, with improving separation, characterization and quantitative techniques, a number of flavonols and their glycosides from the fruit of Vaccinium have been isolated and identified. For example, Zhang et al., identified 12 flavonol glycosides, quercetin-3- $O$ - $\beta$-D-glucuronide (55), quercetin-3- $O$ - $\beta$-D-glucuronide $\mathrm{Me}$ ester (56), quercetin-3- 
Table 2. The Names, Sources, and References of Compounds 42-86

\begin{tabular}{|c|c|c|c|}
\hline No. & Name & Source & References \\
\hline 42 & Chrysin & V. bracteatum (leaves) & [73-75] \\
\hline \multirow[t]{2}{*}{43} & Apigenin & V. bracteatum (leaves) & [73-75] \\
\hline & & V. myrtillus (fruit) & {$[22]$} \\
\hline \multirow[t]{2}{*}{44} & Luteolin & V. bracteatum (leaves) & [73-75] \\
\hline & & V. myrtillus (fruit) & [102] \\
\hline 45 & Chrysoriol & V. myrtillus (fruit) & {$[22]$} \\
\hline \multirow[t]{9}{*}{46} & Kaempferol & V. angustifolium (fruit) & {$[103,104]$} \\
\hline & & V. corymbosum (leaves) & {$[18,46,95]$} \\
\hline & & V. myrtillus (fruit \& leaves) & {$[21,22,46]$} \\
\hline & & V. smallii (leaves) & [72] [49] \\
\hline & & V. bracteatum (leaves) & [73-75] \\
\hline & & V. uliginosum (fruit) & {$[66]$} \\
\hline & & V. vitis-idaea (fruit) & {$[22]$} \\
\hline & & V. $\times$ intermedium (fruit) & {$[22,48]$} \\
\hline & & V. meridionale Swartz (fruit) & [47] \\
\hline \multirow[t]{13}{*}{47} & Quercetin & V. angustifolium (fruit) & {$[103,104]$} \\
\hline & & V. corymbosum (leaves) & {$[18,46,95]$} \\
\hline & & V. koreanum (leaves) & {$[54]$} \\
\hline & & V. calycinum (leaves) & {$[53]$} \\
\hline & & V. myrtillus (fruit \& leaves) & {$[21,22,46]$} \\
\hline & & V. reticulatum (leaves) & {$[53]$} \\
\hline & & V. smallii (leaves) & [72] [49] \\
\hline & & V. bracteatum (leaves) & {$[52,56,73,105]$} \\
\hline & & V. meridionale (fruit) & [47] \\
\hline & & V. floribundum (fruit) & {$[68]$} \\
\hline & & V. uliginosum (fruit) & {$[23,40,66,100]$} \\
\hline & & V. vitis-idaea (fruit) & {$[22,66]$} \\
\hline & & V. $\times$ intermedium (fruit) & {$[22,48]$} \\
\hline \multirow[t]{5}{*}{48} & Isorhamnetin & V. macrocarpon (fruit) & [69] \\
\hline & & V. calycinum (leaves) & {$[53]$} \\
\hline & & V. myrtillus (fruit \& leaves) & {$[21,22,46]$} \\
\hline & & $V \cdot$ reticulatum (leaves) & [53] \\
\hline & & $V . \times$ intermedium (fruit) & {$[22,48]$} \\
\hline \multirow[t]{2}{*}{49} & Quertine-3-O-methyl ether & V. calycinum (leaves) & {$[53]$} \\
\hline & & V. reticulatum (leaves) & [53] \\
\hline \multirow[t]{2}{*}{50} & Myricetin & V. angustifolium (fruit) & {$[103,104]$} \\
\hline & & $V$. virgatum $=V$. ashei $($ fruit $)$ & {$[20]$} \\
\hline
\end{tabular}


Table 2. cont...

\begin{tabular}{|c|c|c|c|}
\hline No. & Name & Source & References \\
\hline & & V. corymbosum (leaves) & {$[40]$} \\
\hline & & V. myrtillus (fruit \& leaves) & {$[22]$} \\
\hline & & V. floribundum (fruit) & {$[68]$} \\
\hline & & V. uliginosum (fruit) & {$[40]$} \\
\hline & & V. vitis-idaea (fruit) & {$[67]$} \\
\hline & & $V . \times$ intermedium (fruit) & {$[22,48]$} \\
\hline \multirow[t]{4}{*}{51} & Laricitrin & V. macrocarpon (fruit) & [69] \\
\hline & & V. myrtillus (fruit \& leaves) & {$[21,22,46]$} \\
\hline & & V. uliginosum (fruit) & {$[66]$} \\
\hline & & $V . \times$ intermedium (fruit) & {$[22,48]$} \\
\hline \multirow[t]{4}{*}{52} & Syringetin & $V$. macrocarpon (fruit) & [69] \\
\hline & & V. myrtillus (fruit) & {$[21,22,46]$} \\
\hline & & V. uliginosum (fruit) & {$[23,66]$} \\
\hline & & $V . \times$ intermedium (fruit) & {$[22,48]$} \\
\hline \multirow[t]{14}{*}{53} & Quertine-3- $O$-glucoside & V. macrocarpon (fruit) & [69] \\
\hline & & V. angustifolium (fruit) & {$[103,104]$} \\
\hline & & $V$. virgatum $=V$. ashei $($ fruit $)$ & {$[63]$} \\
\hline & & V. corymbosum (leaves) & {$[37]$} \\
\hline & & V. calycinum (leaves) & {$[53]$} \\
\hline & & V. myrtillus (fruit) & {$[21,22,46]$} \\
\hline & & $V$. reticulatum (leaves) & {$[53]$} \\
\hline & & V. arctostaphylos (leaves) & {$[58,106]$} \\
\hline & & V. smallii (leaves) & [49] \\
\hline & & V. uliginosum (fruit) & {$[23,66]$} \\
\hline & & V. vitis-idaea (fruit) & {$[42,70]$} \\
\hline & & $V . \times$ intermedium (fruit) & {$[22,48]$} \\
\hline & & V. floribundum (fruit) & {$[68]$} \\
\hline & & V. bracteatum (leaves) & {$[51,64]$} \\
\hline \multirow[t]{9}{*}{54} & Quertine-3-O-galactoside & V. macrocarpon (fruit) & {$[65]$} \\
\hline & & V. oxycoccos (fruit) & {$[107]$} \\
\hline & & V. quadripetalis (leaves) & {$[108]$} \\
\hline & & V. angustifolium (fruit) & {$[103,104]$} \\
\hline & & V. corymbosum (leaves) & {$[37]$} \\
\hline & & V. darrowii (fruit) & [109] \\
\hline & & V. calycinum (leaves) & {$[53]$} \\
\hline & & V. myrtillus (fruit \& leaves) & {$[22]$} \\
\hline & & $V$. reticulatum (leaves) & {$[53]$} \\
\hline
\end{tabular}


Table 2. cont...

\begin{tabular}{|c|c|c|c|}
\hline No. & Name & Source & References \\
\hline & & V. smallii (leaves) & {$[49,72]$} \\
\hline & & V. bracteatum (leaves) & {$[51,64]$} \\
\hline & & V. uliginosum (fruit) & {$[23,66]$} \\
\hline & & V. vitis-idaea (fruit) & {$[42,70]$} \\
\hline \multirow[t]{6}{*}{55} & Quertine-3-O-glucuronide & $V$. virgatum $=V$. ashei $($ fruit $)$ & {$[63]$} \\
\hline & & V. myrtillus (fruit) & {$[22]$} \\
\hline & & $V . \times$ intermedium (fruit) & [48] \\
\hline & & V. bracteatum (leaves) & {$[64]$} \\
\hline & & V. uliginosum (fruit) & {$[23,66]$} \\
\hline & & V. vitis-idaea (fruit) & {$[42,70]$} \\
\hline 56 & Quertine-3-O-glucuronide-6"-methyl ether & V. bracteatum (leaves) & {$[64]$} \\
\hline \multirow[t]{5}{*}{57} & Avicularine & V. macrocarpon (fruit) & [69] \\
\hline & & V. koreanum (leaves) & {$[54]$} \\
\hline & & V. myrtillus (fruit) & {$[21,22,46]$} \\
\hline & & V. smallii (leaves) & [72] [49] \\
\hline & & V. vitis-idaea (fruit) & {$[42,70]$} \\
\hline \multirow[t]{4}{*}{58} & Quertine-3-O-arabinofuranoside & $V . \times$ intermedium (fruit) & [48] \\
\hline & & V. macrocarpon (fruit) & [69] [65] \\
\hline & & V. bracteatum (leaves) & [64] \\
\hline & & V. koreanum (leaves) & [54] \\
\hline \multirow[t]{9}{*}{59} & Quertine-3-O-rhamnoside & V. macrocarpon (fruit) & [69] \\
\hline & & V. angustifolium (fruit) & {$[103,104]$} \\
\hline & & $V$. virgatum $=V$. ashei $($ fruit $)$ & [63] \\
\hline & & V. corymbosum (leaves) & {$[37,46,95]$} \\
\hline & & V. myrtillus (fruit) & {$[21,22,46]$} \\
\hline & & V. smallii (leaves) & [49] \\
\hline & & V. bracteatum (leaves) & [64] \\
\hline & & V. vitis-idaea (fruit) & {$[42,70]$} \\
\hline & & $V . \times$ intermedium (fruit) & {$[48]$} \\
\hline \multirow[t]{5}{*}{60} & Quertine-3-O-galactoside & V. macrocarpon (fruit) & {$[4,69]$} \\
\hline & & V. smallii (leaves) & {$[49,72]$} \\
\hline & & V. bracteatum (leaves) & [64] \\
\hline & & V. vitis-idaea (fruit) & {$[59,70]$} \\
\hline & & V. myrtillus (fruit \& leaves) & {$[21,22,46]$} \\
\hline \multirow[t]{3}{*}{61} & Quertine-3-O-arabinoside & V. $\times$ intermedium (fruit) & [48] \\
\hline & & V. vitis-idaea (fruit) & {$[42,70]$} \\
\hline & & V. corymbosum (leaves) & [37] \\
\hline
\end{tabular}


Table 2. cont...

\begin{tabular}{|c|c|c|c|}
\hline No. & Name & Source & References \\
\hline & & V. myrtillus (fruit \& leaves) & {$[21,22,46]$} \\
\hline & & V. macrocarpon (fruit) & [69] \\
\hline \multirow[t]{4}{*}{62} & Quertine-3-O-xyloside & V. vitis-idaea (fruit) & {$[42,70]$} \\
\hline & & $V . \times$ intermedium (fruit) & {$[22]$} \\
\hline & & V. myrtillus (fruit \& leaves) & {$[22]$} \\
\hline & & V. floribundum (fruit) & {$[68]$} \\
\hline 63 & Quertine-6-O-glucoside & V. bracteatum (leaves) & [52] \\
\hline 64 & Chrysoeriol-7-O-glucoside & V. bracteatum (leaves) & {$[64]$} \\
\hline 65 & Flavogadorinin & V. bracteatum (leaves) & {$[64,83,110]$} \\
\hline 66 & Quertine-3-O-(6"-coumaroyl)galactoside & V. macrocarpon (fruit) & [65] \\
\hline 67 & Quertine-3-O-(6"-benzoyl)galactoside & V. macrocarpon (fruit) & {$[65]$} \\
\hline \multirow[t]{2}{*}{68} & $\begin{array}{l}\text { 3-[[4-O-(4-carboxy-3-hydroxy-3-methyl- } \\
\text { 1-oxobutyl)-6-deoxy- } \alpha \text {-L-mannopyranosyl]oxy]-2-(3,4- } \\
\text { dihydroxyphenyl)-5,7-dihydroxy-4H-1-Benzopyran-4-one }\end{array}$ & V. myrtillus (fruit) & {$[57]$} \\
\hline & & V. vitis-idaea (fruit) & [22] \\
\hline \multirow[t]{2}{*}{69} & $\begin{array}{l}\text { 3-[[4-O-(4-carboxy-3-hydroxy-3-methyl- } \\
\text { 1-oxobutyl)-6-deoxy- } \alpha \text {-L-mannopyranosyl] oxy]-5,7-dihydroxy- } \\
\text { 2-(4-hydroxyphenyl)- 4H-1-Benzopyran-4-one }\end{array}$ & V. myrtillus (fruit) & [57] \\
\hline & & V. vitis-idaea (fruit) & [22] \\
\hline 70 & Myricetin-3-O-arabinoside & V. macrocarpon (fruit) & {$[4,69]$} \\
\hline \multirow[t]{4}{*}{71} & Isorhamnetin-3-O-galactoside & V. macrocarpon (fruit) & [69] \\
\hline & & V. myrtillus (fruit \& leaves) & {$[21,22,46]$} \\
\hline & & V. uliginosum (fruit) & {$[23,66]$} \\
\hline & & V. vitis-idaea (fruit) & {$[42,70]$} \\
\hline \multirow[t]{3}{*}{72} & Myricetin-3-O-glucuronide & V. myrtillus (fruit \& leaves) & {$[21,22,46]$} \\
\hline & & V. uliginosum (fruit) & {$[21]$} \\
\hline & & $V . \times$ intermedium (fruit) & {$[68]$} \\
\hline \multirow[t]{4}{*}{73} & Myricetin-3-O-glucoside & V. angustifolium (fruit) & {$[103,104]$} \\
\hline & & V. corymbosum (leaves) & [95] \\
\hline & & V. myrtillus (fruit \& leaves) & {$[21,22,46]$} \\
\hline & & V. vitis-idaea (fruit) & {$[67]$} \\
\hline \multirow[t]{2}{*}{74} & Myricetin-3-O-xyloside & V. macrocarpon (fruit) & [69] \\
\hline & & V. myrtillus (fruit \& leaves) & [22] \\
\hline \multirow[t]{2}{*}{75} & Laricitrin-3-O-glucuronide & V. myrtillus (fruit) & {$[21,22,46]$} \\
\hline & & $V . \times$ intermedium (fruit) & {$[68]$} \\
\hline \multirow[t]{3}{*}{76} & Isorhamnetin-3-O-glucoside & V. uliginosum (fruit) & {$[23,66]$} \\
\hline & & V. myrtillus (fruit \& leaves) & {$[22]$} \\
\hline & & V. bracteatum (leaves) & {$[64]$} \\
\hline
\end{tabular}


Table 2. cont...

\begin{tabular}{|c|c|c|c|}
\hline No. & Name & Source & References \\
\hline & & V. vitis-idaea (fruit) & [70] \\
\hline \multirow[t]{4}{*}{77} & Syringetin-3-O-glucoside & V. corymbosum (leaves) & {$[46,95]$} \\
\hline & & V. myrtillus (fruit \& leaves) & {$[21,22,46]$} \\
\hline & & V. uliginosum (fruit) & {$[23,66]$} \\
\hline & & V. vitis-idaea (fruit) & {$[70]$} \\
\hline \multirow[t]{5}{*}{78} & Kaempferol-3-O-glucoside & V. angustifolium (fruit) & {$[103,104]$} \\
\hline & & V. corymbosum (leaves) & {$[46,95]$} \\
\hline & & $V$. arctostaphylos (leaves) & {$[58,111]$} \\
\hline & & V. myrtillus (fruit) & {$[21]$} \\
\hline & & V. vitis-idaea (fruit) & {$[70]$} \\
\hline \multirow[t]{2}{*}{79} & Kaempferol-3-O-rhamnoside & V. arctostaphylos (leaves) & {$[58,111]$} \\
\hline & & V. vitis-idaea (fruit) & {$[42]$} \\
\hline \multirow[t]{3}{*}{80} & Myricetin-3-O-galactoside & V. macrocarpon (fruit) & [69] \\
\hline & & V. myrtillus (fruit) & {$[21]$} \\
\hline & & V. uliginosum (fruit) & {$[23,66]$} \\
\hline \multirow[t]{2}{*}{81} & Isorhamnetin-3-O-xyloside & V. macrocarpon (fruit) & [69] \\
\hline & & V. myrtillus (fruit) & {$[21,62]$} \\
\hline 82 & Orientin & V. bracteatum (leaves) & [64] \\
\hline 83 & Vitexina & V. bracteatum (leaves) & [64] \\
\hline 84 & Chrysoeriol-7-O-(6"-O-p-coumaroyl)- glucopyranoside & V. bracteatum (leaves) & {$[64]$} \\
\hline 85 & Myricetin-3-O-digalactoside & V. macrocarpon (fruit) & [69] \\
\hline \multirow[t]{10}{*}{86} & Quertine-3- $O$-rutinoside & V. bracteatum (leaves) & {$[64]$} \\
\hline & & V. angustifolium (fruit) & {$[103,104]$} \\
\hline & & $V$. virgatum $=V$. ashei $($ fruit $)$ & [63] \\
\hline & & V. corymbosum (leaves) & [95] \\
\hline & & V. darrowii (fruit) & [109] \\
\hline & & V. myrtillus (fruit) & {$[21,62]$} \\
\hline & & V. arctostaphylos (leaves) & [111] \\
\hline & & V. uliginosum (fruit) & {$[23,66]$} \\
\hline & & V. vitis-idaea (fruit) & {$[70]$} \\
\hline & & $V . \times$ intermedium (fruit) & {$[68]$} \\
\hline
\end{tabular}

$O$ - $\alpha$-L-rhamnoside (59), quercetin-3- $O$ - $\beta$-D-galactopyranoside (60), quercetin-3- $O-\alpha$-L-arabinopyranoside (61), chrysoeriol-7- $O$ - $\beta$-D-glucopyranoside (64), flavogadorinin (65), isorhamnetin-3-O- $\beta$-D-glucopyranoside (76), isoorientin (81), orientin (82), vitexin (83), and chrysoeriol-7- $O-\left(6^{\prime \prime}-O\right.$ $p$-coumaroyl)- $\beta$-D- glucopyranoside (84) from the leaves of $V$. bracteatum. Compounds 56, 64, 65 and 84 were isolated from the Vaccinium for the first time, and compounds $\mathbf{5 5}, \mathbf{5 9}$, 60, 61, 76, 82 and 83 were isolated from this plant for the first time. The $p$-coumaroylglucoside of compound 84 may gradually hydrolyze in the extract to give the free acid. Compounds orientin (82) and vitexin (83) are 8-Cglucosylflavones in nature. The UV/Vis spectrum of the aglycone, 8-C-glucosylflavone, has a $\lambda_{\max }$ at $541 \mathrm{~nm}$ in $\mathrm{MeOH} / \mathrm{HCl}$, in between that of cyanidin (at $535 \mathrm{~nm}$ ) and delphinidin (at $546 \mathrm{~nm}$ ). Therefore, 8-C-glucosylflavone appears to represent another blueing factor of flower color in addition to hydroxylation of the B-ring, acylation, co- 
pigmentation and metal complexation [64], [94]. Vvedenskaya et al. isolated 22 flavonoids by UV/vis and mass spectra analyses from cranberry powder. Among them, six new constituents not previously reported in cranberry or in cranberry products were determined through NMR spectroscopy to be quercetin-3- $\beta$-glucoside (53), quercetin-3$\alpha$-arabinopyranoside (61), quercetin-3-O- (6"-p-coumaroyl)$\beta$-galactoside (66), quercetin-3-O-(6"-benzoyl)- $\beta$-galactoside (67) myricetin-3- $\beta$-xylopyranoside (74), and 3 -methoxyquercetin-3- $\alpha$-xylopyranoside (81). Compounds 66 and 67 represent a new class of cranberry flavonol compounds with three conjugated components consisting of a flavonol, sugar, and carboxylic acid (benzoic or hydroxycinnamic acids). This is also the first report identifying quercetin-3arabinoside in both furanose and pyranose forms in cranberry [69].

In addition, some flavonols and their derivatives were also isolated from the $V$. vitis-idaea. These compounds include kaempferol (46), quercetin (47), quercetin 3-O- $\beta$-Dgalactoside (54), quercetin $3-O-\alpha$-L-arabinoside (61), and quercetin 3 - $O$ - $\alpha$-L-rhamnopyranosyl $(1 \rightarrow 6)-\beta$-D-glucopyranoside $(\mathbf{8 5})[22,66]$.<smiles>O=c1cc(-c2ccccc2)oc2cc(O)cc(O)c12</smiles>

42<smiles>COc1ccc(-c2cc(=O)c3c(O)cc(O)cc3o2)cc1O</smiles>

45<smiles>COc1cc(-c2oc3cc(O)cc(O)c3c(=O)c2O)ccc1O</smiles>

48<smiles>COc1cc(-c2oc3cc(O)cc(O)c3c(=O)c2O)cc(O)c1O</smiles>

51<smiles>O=c1c(O[C@@H]2O[C@H](CO)[C@@H](O)[C@H](O)[C@H]2O)c(-c2ccc(O)c(O)c2)oc2cc(O)cc(O)c12</smiles>

54<smiles>O=c1cc(-c2ccc(O)cc2)oc2cc(O)cc(O)c12</smiles>

43<smiles>O=c1c(O)c(-c2ccc(O)cc2)oc2cc(O)cc(O)c12</smiles><smiles>COc1c(-c2ccc(O)c(O)c2)oc2cc(O)cc(O)c2c1=O</smiles>

49<smiles>COc1cc(-c2oc3cc(O)cc(O)c3c(=O)c2O)cc(OC)c1O</smiles>

52<smiles>O=C(O)[C@H]1O[C@@H](Oc2c(-c3ccc(O)c(O)c3)oc3cc(O)cc(O)c3c2=O)[C@H](O)[C@H](O)[C@@H]1O</smiles>

55<smiles>O=c1cc(-c2ccc(O)c(O)c2)oc2cc(O)cc(O)c12</smiles>

44<smiles>O=c1c(O)c(-c2ccc(O)c(O)c2)oc2cc(O)cc(O)c12</smiles><smiles>O=c1c(O)c(-c2cc(O)c(O)c(O)c2)oc2cc(O)cc(O)c12</smiles>

50<smiles>O=c1c(O[C@@H]2O[C@H](CO)[C@@H](O)[C@H](O)[C@H]2O)c(-c2ccc(O)c(O)c2)oc2cc(O)cc(O)c12</smiles><smiles>CC(=O)[C@H]1O[C@H](Oc2c(-c3ccc(O)c(O)c3)oc3cc(O)cc(O)c3c2=O)[C@H](O)[C@@H](O)[C@@H]1O</smiles>

56 
<smiles>O=c1c(O[C@@H]2O[C@H](CO)[C@@H](O)[C@H]2O)c(-c2ccc(O)c(O)c2)oc2cc(O)cc(O)c12</smiles>

57<smiles>O=c1c(O[C@@H]2OC[C@@H](O)[C@H](O)[C@H]2O)c(-c2ccc(O)c(O)c2)oc2cc(O)cc(O)c12</smiles>

60<smiles>O=c1c(O[C@@H]2O[C@H](CO)[C@@H](O)[C@H]2O)c(-c2ccc(O)c(O)c2)oc2cc(O)cc(O)c12</smiles>

58<smiles>O=c1c(O[C@@H]2OC[C@@H](O)[C@H](O)[C@H]2O)c(-c2ccc(O)c(O)c2)oc2cc(O)cc(O)c12</smiles>

61<smiles>C[C@@H]1O[C@H](Oc2c(-c3ccc(O)c(O)c3)oc3cc(O)cc(O)c3c2=O)[C@H](O)[C@H](O)[C@@H]1O</smiles>

59<smiles>O=c1c(O[C@@H]2OC[C@@H](O)[C@H](O)[C@H]2O)c(-c2ccc(O)c(O)c2)oc2cc(O)cc(O)c12</smiles>

62<smiles></smiles>

63

64

65

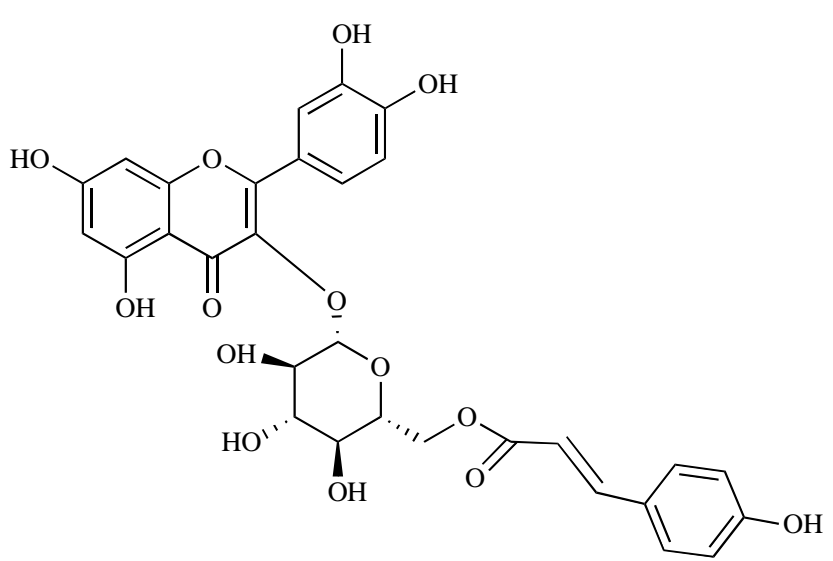

66<smiles>O=C(OC[C@H]1OC[C@@H](O)[C@H](O)[C@@H]1O)c1ccc(O)cc1</smiles>

67 
<smiles>C[C@H]1O[C@@H](Oc2c(-c3ccc(O)c(O)c3)oc3cc(O)cc(O)c3c2=O)[C@H](O)[C@H](O)[C@@H]1OC(=O)CC(C)(O)CC(=O)O</smiles>

68<smiles>COc1cc(-c2oc3cc(O)cc(O)c3c(=O)c2O[C@@H]2O[C@H](CO)[C@@H](O)[C@H](O)[C@H]2O)ccc1O</smiles>

71<smiles>O=c1c(O[C@@H]2OC[C@@H](O)[C@H](O)[C@H]2O)c(-c2cc(O)c(O)c(O)c2)oc2cc(O)cc(O)c12</smiles>

74<smiles>COc1cc(-c2oc3cc(O)cc(O)c3c(=O)c2O[C@@H]2O[C@H](CO)[C@@H](O)[C@H](O)[C@H]2O)cc(OC)c1O</smiles>

77<smiles>O=c1c(O[C@@H]2O[C@H](CO)[C@@H](O)[C@H](O)[C@H]2O)c(-c2cc(O)c(O)c(O)c2)oc2cc(O)cc(O)c12</smiles><smiles>C[C@@H]1O[C@H](Oc2c(-c3ccc(O)cc3)oc3cc(O)cc(O)c3c2=O)[C@H](O)[C@H](O)[C@@H]1OC(=O)CC(C)(O)CC(=O)O</smiles>

69<smiles>O=C(O)[C@H]1O[C@@H](Oc2c(-c3cc(O)c(O)c(O)c3)oc3cc(O)cc(O)c3c2=O)[C@H](O)[C@@H](O)[C@@H]1O</smiles>

72<smiles>COc1cc(-c2oc3cc(O)cc(O)c3c(=O)c2O[C@@H]2O[C@H](C(=O)O)[C@@H](O)[C@H](O)[C@H]2O)cc(O)c1O</smiles>

75<smiles>O=c1c(O[C@@H]2O[C@H](CO)[C@@H](O)[C@H](O)[C@H]2O)c(-c2ccc(O)cc2)oc2cc(O)cc(O)c12</smiles>

78<smiles>COc1cc(-c2oc3cc(O)cc(O)c3c(=O)c2OC2OC[C@@H](O)[C@H](O)[C@H]2O)ccc1O</smiles>

81<smiles>O=c1c(O[C@@H]2OC[C@@H](O)[C@H](O)[C@H]2O)c(-c2cc(O)c(O)c(O)c2)oc2cc(O)cc(O)c12</smiles>

70<smiles>O=c1c(OC2O[C@@H](CO)[C@H](O)[C@@H](O)[C@H]2O)c(-c2cc(O)c(O)c(O)c2)oc2cc(O)cc(O)c12</smiles>

73<smiles>COc1cc(-c2oc3cc(O)cc(O)c3c(=O)c2OC2O[C@H](CO)[C@@H](O)[C@H](O)[C@H]2O)ccc1O</smiles>

76<smiles>C[C@@H]1O[C@H](Oc2c(-c3ccc(O)cc3)oc3cc(O)cc(O)c3c2=O)[C@H](O)[C@@H](O)[C@@H]1O</smiles>

79<smiles>O=c1cc(-c2ccc(O)c(O)c2)oc2c(C3O[C@H](CO)[C@@H](O)[C@H](O)[C@H]3O)c(O)cc(O)c12</smiles>

82 
<smiles>O=c1cc(-c2ccc(O)cc2)oc2c(C3O[C@H](CO)[C@@H](O)[C@H](O)[C@H]3O)c(O)cc(O)c12</smiles>

83<smiles></smiles>

Flavan-3-ols and Proanthocyanidins

Proanthocyanidins, also known as procyanidins, are a class of flavanols. Proanthocyanidin are essentially polymer chains of flavonoids such as flavan-3-ols (catechins). The composition and content of procyanidins have been studied in common plant foods $[112,113]$. The universal B-type procyanidins have a single link between structural units of catechins, whereas the rare A-type procyanidins are double linked. This unique double linked chain structure of flavonoids aroused special interest, because it was suspected to contribute to antiadhesion activity against bacteria and to the antiviral effects of these food products. Cultivated cranberry ( $V$. macrocarpon Aiton) and wild lingonberry contain both A- and B-type procyanidins, whereas primary B-type procyanidins were identified in wild ( $V$. angustifolium Aiton) and cultivated blueberries ( $V$. corymbosum and $V$. virgatum) [114-118]. To data, four flavan-3-ols and 24 proanthocyanidins have been isolated and identified from the genus Vaccinium (Table 3). Recently some other reports have studied the composition and contents of proanthocyanidin of Vaccinium species using HPLC-MS after extraction from the leaves $[119,120]$.

\section{BIOLOGICAL ACTIVITIES}

Vaccinium species are rich in anthocyanins and flavonoids. Many reports have suggested that these compounds exhibit a wide range of biological activities, e.g., antioxidant, anti-inflammatory and anticancer effects (Table 4 and 5) [78, 121, 122]. Thus, they are assumed to promote health by protecting one from various degenerative diseases and diabetes as well as enhancing visual function and slowing the progression of neurological disorders. Consumption of flavonoid-rich plant foods has been claimed to protect against cardiovascular diseases and certain cancers, such as lung cancer [123]. It is known that the oxidation of low density lipoproteins (LDL) is associated with cardiovascular diseases, and thus flavonoids, compounds possessing antioxidant activity, are postulated to have potential benefits in the prevention of these diseases $[124,125]$.

\section{BIOSYNTHESIS PATHWAYS OF ANTHOCYANINS AND FLAVONOIDS (FIG. 3) [172-173]}

As anthocyanins and flavonoids shared a basic $\mathrm{C}_{6}-\mathrm{C}_{3}-\mathrm{C}_{6}$ skeleton system, their biosynthetic precursor was thus proposed to be cinnamoyl-CoA starter unit. The chain can be extended to give naringenin chalcone by using three molecules of malonyl-CoA. Then the naringenin chalcone generates aromatic rings through the chalcone isomerase (CHI). Chalcones act as precursors for a vast range of flavonoid derivatives found throughout the plant kingdom. Most contain a six-membered heterocyclic ring, formed by Michael-type nucleophilic attack of a phenol group on to the unsaturated ketone giving a flavanone. This isomerization can occur chemically, acid conditions favouring the flavanone and basic conditions the chalcone, but in nature the reaction is enzyme catalysed and stereospecific, resulting in formation of a single flavanone enantiomer. Flavanones can then give rise to many variants on this basic skeleton, e.g. flavones, flavonols, and anthocyanidins. Modifications to the hydroxylation patterns in the two aromatic rings may occur, generally at the flavanone 
Table 3. The Names, Sources, and References of Compounds 87-116

\begin{tabular}{|c|c|c|c|}
\hline No. & Name & Source & References \\
\hline \multirow[t]{3}{*}{87} & (-)-epicatechin & V. virgatum $=V$. ashei $($ leaves $)$ & {$[63]$} \\
\hline & & V. vitis-idaea (whole plant) & [117] \\
\hline & & V. vitis-idaea (fruit) & {$[42]$} \\
\hline \multirow[t]{3}{*}{88} & $(+)$-catechin & V. virgatum $=V$. ashei $($ leaves $)$ & {$[63]$} \\
\hline & & V. vitis-idaea (whole plant) & {$[117]$} \\
\hline & & V. vitis-idaea (fruit) & {$[42]$} \\
\hline \multirow[t]{2}{*}{89} & (-)-epigallocatechin & V. virgatum $=V$. ashei $($ leaves $)$ & [63] \\
\hline & & V. vitis-idaea (whole plant) & [117] \\
\hline \multirow[t]{2}{*}{90} & (+)-gallocatechin & $V$. virgatum $=V$. ashei (leaves) & [63] \\
\hline & & V. vitis-idaea (whole plant) & [117] \\
\hline 91 & Mururin A & $V$. virgatum $=V$. ashei $($ leaves $)$ & [63] \\
\hline 92 & Mururin B & $V$. virgatum $=V$. ashei (leaves) & [63] \\
\hline 93 & Vaccinin A & V. virgatum $=V$. ashei (leaves) & [63] \\
\hline 94 & Cinchonain Ia & $V$. virgatum $=V$. ashei (leaves) & [63] \\
\hline 95 & Cinchonain Ib & $V$. virgatum $=V$. ashei (leaves) & [63] \\
\hline 96 & $\begin{array}{l}{[2 \mathrm{R}-(2 \alpha, 3 \beta, 10 \beta)]-2,10 \text {-bis(3,4-dihydroxy phenyl)-3,44,9,10-tetrahydro- }} \\
\text { 3,5-dihydroxy- } \\
\text { 2H,8H-benzo[1,2-b:3,4-b']dipyran-8-one }\end{array}$ & $V$. virgatum $=V$. ashei $($ leaves $)$ & {$[63]$} \\
\hline 97 & Kandelin A-2 & $V$. virgatum $=V$. ashei (leaves) & [63] \\
\hline 98 & Cinchonain IIb & $V$. virgatum $=V$. ashei (leaves) & [63] \\
\hline 99 & Kandelin A-1 & V. virgatum $=V$. ashei $($ leaves $)$ & [63] \\
\hline 100 & Cinchonain IIa & $V$. virgatum $=V$. ashei $($ leaves) & {$[63]$} \\
\hline 101 & Procyanidin B-3 & V. vitis-idaea (whole plant) & {$[117]$} \\
\hline 102 & Procyanidin B-1 & V. virgatum $=V$. ashei $($ leaves $)$ & {$[63]$} \\
\hline 103 & Procyanidin B-2 & V. virgatum $=V$. ashei $($ leaves $)$ & [63] \\
\hline 104 & Procyanidin B-7 & V. vitis-idaea (whole plant) & [117] \\
\hline 105 & Procyanidin C-1 & V. virgatum $=V$. ashei $($ leaves $)$ & {$[63]$} \\
\hline 106 & Proanthocyanidin A-2 & V. vitis-idaea (whole plant) & {$[117]$} \\
\hline 107 & Proanthocyanidin A-1 & V. vitis-idaea (whole plant) & [117] \\
\hline 108 & $\begin{array}{l}\text { Epicatechin- }(4 \beta \rightarrow 8,2 \beta \rightarrow 7) \text {-epicatechin- } \\
\qquad(4 \alpha \rightarrow 8) \text {-epicatechin }\end{array}$ & V. virgatum $=V$. ashei $($ leaves $)$ & {$[63]$} \\
\hline 109 & (+)-gallocatechin & V. vitis-idaea (whole plant) & [117] \\
\hline 110 & $\begin{array}{l}\text { Epicatechin- }(4 \beta \rightarrow 8,2 \beta \rightarrow 7) \text {-epicatechin- } \\
\qquad(4 \alpha \rightarrow 8) \text {-catechin }\end{array}$ & $V$. virgatum $=V$. ashei (leaves) & [63] \\
\hline 111 & Cinnamtannin D1 & V. vitis-idaea (whole plant) & [117] \\
\hline 112 & Cinnamtannin B1 & V. vitis-idaea (whole plant) & {$[117]$} \\
\hline 113 & Cinnamtannin D2 & V. vitis-idaea (whole plant) & {$[117]$} \\
\hline 114 & Cinnamtannin B2 & V. vitis-idaea (whole plant) & [117] \\
\hline 115 & $\begin{array}{l}\text { Epicatechin- }(4 \beta \rightarrow 8,2 \beta \rightarrow 7) \text { - epicatechin- } \\
\quad(4 \alpha \rightarrow 8) \text {-epicatechin- }(4 \beta \rightarrow 8) \text {-catechin }\end{array}$ & V. virgatum $=V$. ashei $($ leaves $)$ & {$[63]$} \\
\hline 116 & $\begin{array}{l}\text { Epicatechin- }(4 \beta \rightarrow 8,2 \beta \rightarrow 7) \text {-epicatechin- } \\
(4 \alpha \rightarrow 8) \text {-epicatechin- }(4 \beta \rightarrow 6) \text {-catechin }\end{array}$ & V. virgatum $=V$. ashei $($ leaves $)$ & [63] \\
\hline
\end{tabular}


<smiles>Oc1cc(O)c2c(c1)O[C@H](c1ccc(O)c(O)c1)[C@H](O)C2</smiles><smiles>Oc1cc(O)c2c(c1)O[C@H](c1ccc(O)c(O)c1)[C@H](O)C2</smiles><smiles>Oc1cc(O)c2c(c1)O[C@H](c1cc(O)c(O)c(O)c1)[C@H](O)C2</smiles>

88

89<smiles></smiles><smiles></smiles>

93<smiles>O=C1C[C@H](c2ccc(O)c(O)c2)c2c(cc(O)c3c2O[C@H](c2ccc(O)c(O)c2)[C@H](O)C3)O1</smiles>

96<smiles>O=C1C[C@H](c2ccc(O)c(O)c2)c2c(cc(O)c3c2O[C@H](c2ccc(O)c(O)c2)[C@H](O)C3)O1</smiles>

94<smiles>Oc1cc(O)c2c(c1)O[C@@]1(c3ccc(O)c(O)c3)O[C@@H]2[C@H](c2c(O)cc(O)c3c2O[C@H](c2ccc(O)c(O)c2)[C@@H](O)C3)[C@H]1O</smiles>

97<smiles>O=C1C[C@H](c2ccc(O)c(O)c2)c2c(cc(O)c3c2O[C@H](c2ccc(O)c(O)c2)[C@H](O)C3)O1</smiles>

95

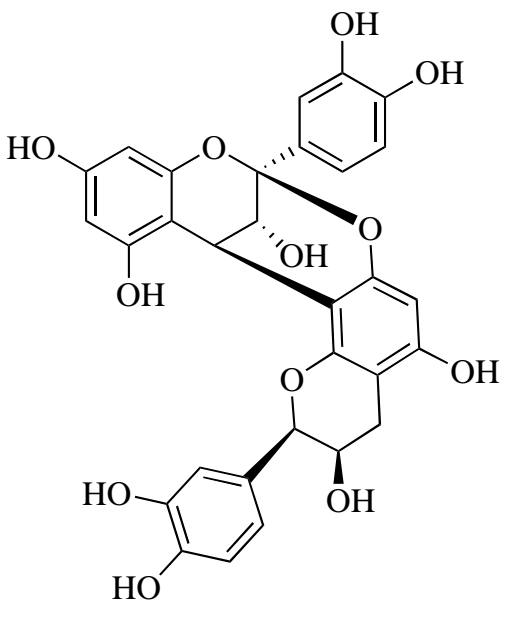

98 
<smiles>Oc1cc(O)c2c(c1)O[C@H](c1ccc(O)c(O)c1)[C@H](O)[C@H]2c1c(O)cc(O)c2c1O[C@H](c1ccc(O)c(O)c1)[C@H](O)C2</smiles>

99<smiles>Oc1cc(O)c2c(c1)O[C@H](c1ccc(O)c(O)c1)[C@H](O)[C@H]2c1c(O)cc(O)c2c1O[C@H](c1ccc(O)c(O)c1)[C@H](O)C2</smiles>

100<smiles>Oc1cc(O)c2c(c1)O[C@H](c1ccc(O)c(O)c1)[C@H](O)[C@H]2c1c(O)cc(O)c2c1O[C@H](c1ccc(O)c(O)c1)[C@H](O)C2</smiles>

101<smiles>Oc1cc(O)c2c(c1)O[C@H](c1ccc(O)c(O)c1)[C@H](O)[C@H]2c1c(O)cc2c(c1O)C[C@@H](O)[C@H](c1ccc(O)c(O)c1)O2</smiles>

102<smiles>O=C1C[C@H](c2ccc(O)c(O)c2)c2c(cc(O)c3c2O[C@H](c2ccc(O)c(O)c2)[C@H](O)[C@H]3c2c(O)cc(O)c3c2OC(c2ccc(O)c(O)c2)[C@H](O)C3O)O1</smiles>

103<smiles>O=C1C[C@H](c2ccc(O)c(O)c2)c2c(cc(O)c3c2O[C@H](c2ccc(O)c(O)c2)[C@H](O)[C@H]3c2c(O)cc(O)c3c2OC(c2ccc(O)c(O)c2)[C@H](O)C3O)O1</smiles>

104<smiles>Oc1cc(O)c2c(c1)O[C@H](c1cc(O)cc(O)c1[C@H]1c3c(O)cc(O)c([C@H]4c5c(O)cc(O)cc5O[C@H](c5ccc(O)c(O)c5)[C@@H]4O)c3O[C@H](c3ccc(O)c(O)c3)[C@@H]1O)[C@H](O)C2</smiles>

105<smiles>O=C1C[C@H](c2ccc(O)c(O)c2)c2c(cc(O)c3c2O[C@H](c2ccc(O)c(O)c2)[C@H](O)[C@H]3c2c(O)cc(O)c3c2OC(c2ccc(O)c(O)c2)[C@H](O)C3O)O1</smiles>

106<smiles>O=C1C[C@H](c2ccc(O)c(O)c2)c2c(cc(O)c3c2O[C@H](c2ccc(O)c(O)c2)[C@H](O)[C@H]3c2c(O)cc(O)c3c2OC(c2ccc(O)c(O)c2)[C@H](O)C3O)O1</smiles>

107 
<smiles>Oc1cc(O)c2c(c1)O[C@]1(c3ccc(O)c(O)c3)Oc3c(O)cc(O)c4c3O[C@H](c3ccc(O)c(O)c3)[C@H](O4)[C@H]2[C@H]1c1c(O)cc(O)c2c1O[C@H](c1ccc(O)c(O)c1)[C@H](O)C2</smiles>

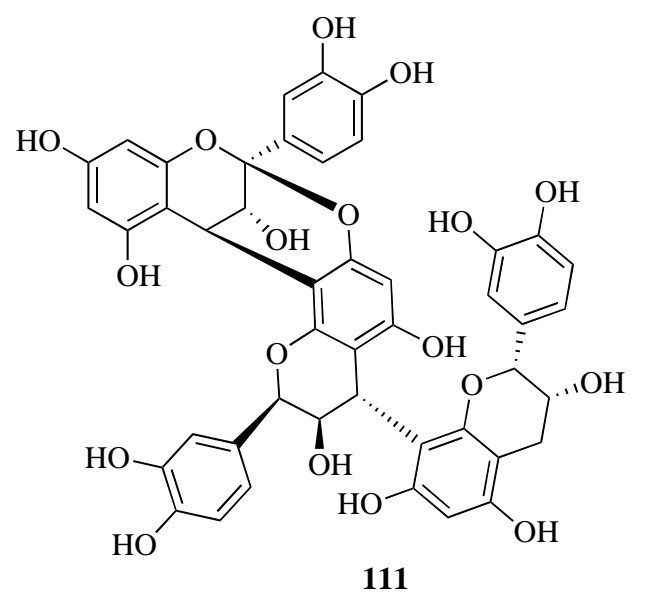

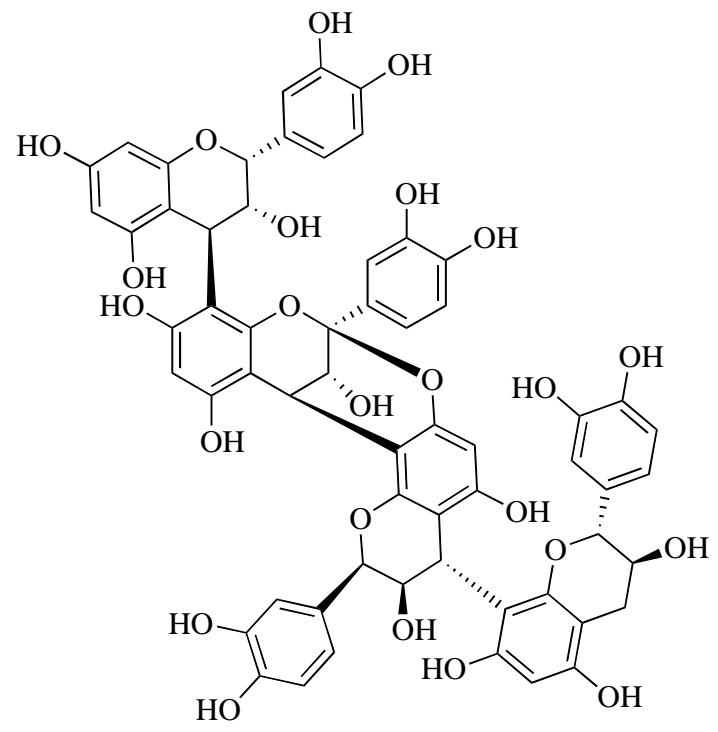

113

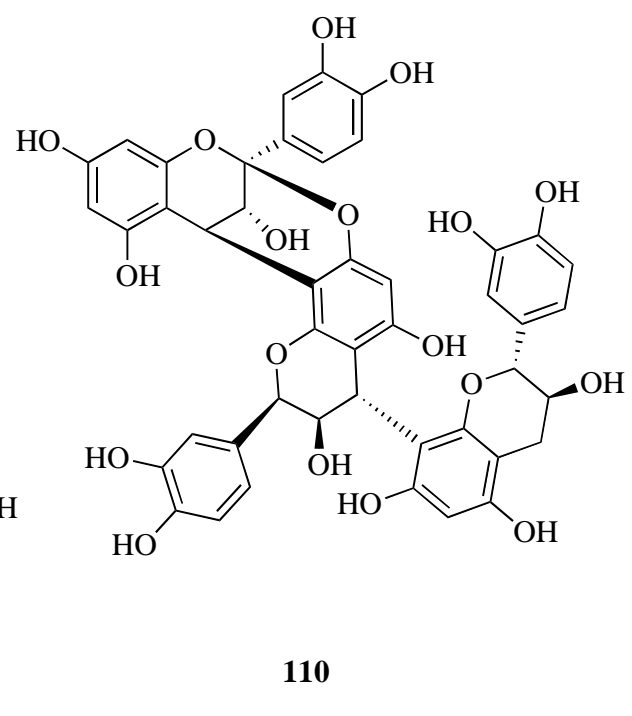

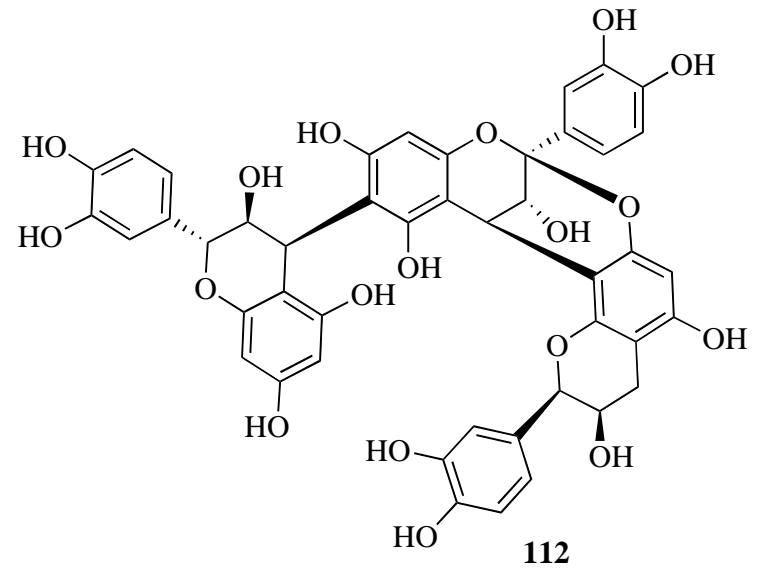

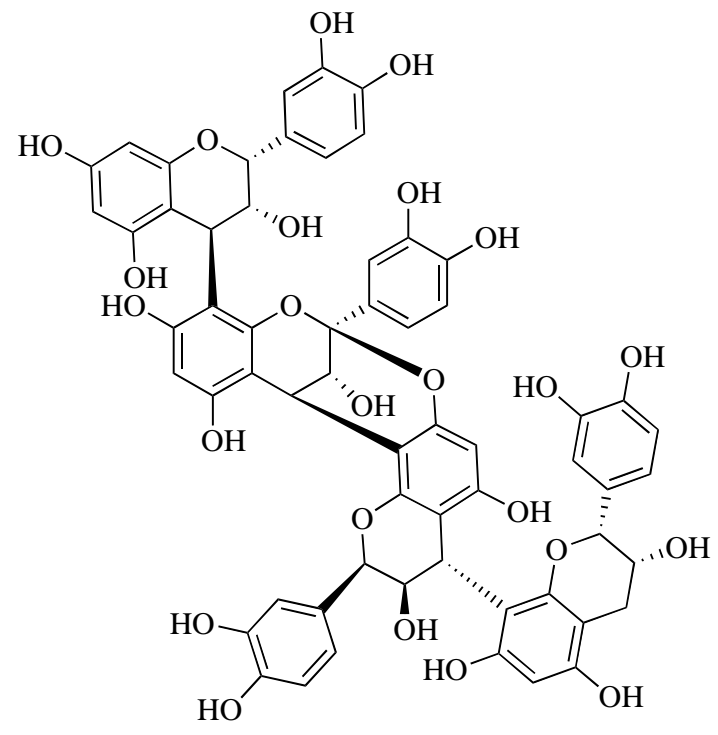




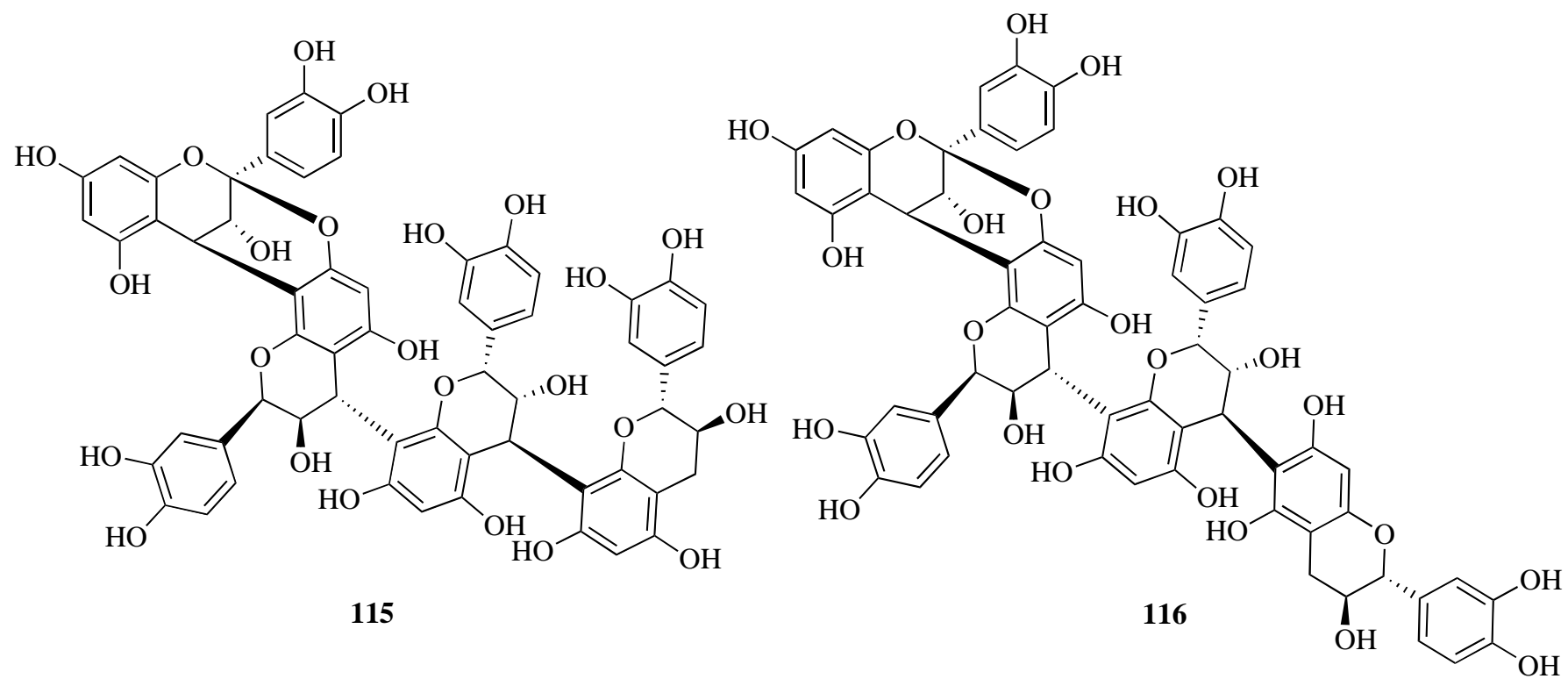

Table 4. Biological Activities of Vaccinium L. as Revealed by In Vitro Studies

\begin{tabular}{|c|c|c|}
\hline Bioactivities & Description & References \\
\hline \multirow[t]{5}{*}{ Antioxidant Activities } & $\begin{array}{l}\text { Inhibited over } 90 \% \text { of the formation of methyl linoleate hydroperoxides by cranberry } \\
\text { phenolics at concentration as low as } 500 \mu \mathrm{g} / \mathrm{mL}\end{array}$ & [126] \\
\hline & Inhibited lipid peroxidation, as well as protein and lipid oxidation in liposomes & [127-129] \\
\hline & $\begin{array}{l}\text { Showed to possess radical scavenging capacity in various in vitro models using assays of } \\
\text { the oxygen radical absorbance capacity (ORAC), the total oxidant scavenging capacity } \\
\text { (TOSC), and the free radical scavenging activity against 2,2-diphenyl-1-picrylhydrazyl } \\
\text { (DPPH) radical as well as antioxidant capacities inhibiting oxidation of methyl linoleate, } \\
\text { liposomers, and human LDL. The most striking antioxidant activity is cyaniding 3- } \\
\text { galactose. DPPH assays showed that it was more effevtive than flavonol glycoside and } \\
\text { the standards in scavenging free radicals, with an } \mathrm{EC}_{50} \text { of } 7.7 \mu \mathrm{M} \text { compared to } 17.3 \mu \mathrm{M} \\
\text { for myricetin 3-arabinoside and } 30 \mu \mathrm{M} \text { for trolox standard }\end{array}$ & {$[4,130,131]$} \\
\hline & Showed neuro and mitochondrio-protective effect & [134] \\
\hline & $\begin{array}{l}\text { Showed antioxidative activity under the used assay conditions towards prevention of } \\
\text { oxidative DNA damage }\end{array}$ & {$[135]$} \\
\hline \multirow[t]{4}{*}{ Cytotoxicities } & Inhibited proliferation of MCF-7, MDA-MB-231 and MDA-MB-435 breast cancer cells & {$[4,8,126,127]$} \\
\hline & $\begin{array}{l}\text { Inhibited the induction of ornithine decarboxylase, an enzyme involved in tumor } \\
\text { proliferation, and induce quinine reductase, an enzyme that can inactivate certain } \\
\text { carcinogens }\end{array}$ & {$[136]$} \\
\hline & Exhibited the human colon tumor cell lines Caco-2, HT-116, and HT-29 & {$[10,137,138]$} \\
\hline & Exhibited the anticancer effects on DLD- 1 and COLO205 cells & [139] \\
\hline
\end{tabular}


Table 4. cont...

\begin{tabular}{|c|c|c|}
\hline Bioactivities & Description & References \\
\hline \multirow[t]{5}{*}{ Antimicrobial Activities } & Exhibited selective antibacterial activity against the bacterial strains & [143-146] \\
\hline & Prevented the urinary tract infections & [143] \\
\hline & $\begin{array}{c}\text { Showed potential inhibitory effect against bacteria involved in dental caries and } \\
\text { periodontal diseases }\end{array}$ & {$[147,148]$} \\
\hline & $\begin{array}{l}\text { Exhibited potent biological activity by inhibiting adherence of uropathogenic isolates of } \\
\text { P-fimbriated Escherichia coli bacteria to cellular surfaces containing } \alpha \text {-Gal }(1 \rightarrow 4)-\beta \text {-Gal } \\
\text { receptor sequences similar to those on epithelial cells in the urinary tract }\end{array}$ & {$[149,150]$} \\
\hline & $\begin{array}{l}\text { Inhibit the adsorption of phage T4 to its bacterial host cells and prevented the replication } \\
\text { of rotavirus in its monkey kidney (MA-104) host cells }\end{array}$ & [151] \\
\hline \multirow{2}{*}{$\begin{array}{l}\text { Antidiabetic } \\
\text { Activities }\end{array}$} & Cinchonain Ib has an insulinotropic effect and can be used for managing type 2 diabetes & [152] \\
\hline & $\begin{array}{l}\text { Extracts of the Canadian blueberry contain active principles with insulin-like and } \\
\text { glitazone-like properties, while conferring protection against glucose toxicity }\end{array}$ & [153] \\
\hline $\begin{array}{l}\text { Antinociceptive } \\
\text { Activities }\end{array}$ & Extract from V. corymbosum displayed significantly antinociceptive activity & [154] \\
\hline $\begin{array}{l}\text { Antiinflammatory } \\
\text { Activities }\end{array}$ & $\begin{array}{l}\text { Extract from } V . \text { corymbosum, } V . \text { vitis-idaea, and } V \text {. microcarpon displayed significantly } \\
\text { anti-inflammatory activity }\end{array}$ & {$[154,155]$} \\
\hline Antiviral Activities & Extract from blueberry displayed significantly antinociceptive activity & [156] \\
\hline $\begin{array}{l}\text { Vasoprotective } \\
\text { Activities }\end{array}$ & $\begin{array}{l}\text { Anthocyanin-rich extract from bilberry showed direct vasoactive and vasoprotective } \\
\text { properties }\end{array}$ & [157] \\
\hline
\end{tabular}

Table 5. Biological Activities of Vaccinium L. as Revealed by In Vivo Studies

\begin{tabular}{|c|c|c|}
\hline Model & Description & References \\
\hline Rats & $\begin{array}{c}\text { Flavonoids from fruit residues of } V \text {. vitis-idaea could lower serum uric acid and protect kidney in adenine-reduced } \\
\text { hyperuricemia }\end{array}$ & [132] \\
\hline Mice & Anthocyanins from bilberry showed protective effect on gastric ulcer & [159] \\
\hline Mice & $\begin{array}{l}\text { The bilberry fruit extract, in a dose dependent manner, induced the resolution of liver fibrosis by decreasing } \\
\text { oxidative stress and inactivating HSCs via down-regulation of fibrogenic cytokines, TGF- } \beta 1 \text { and TNF- } \alpha\end{array}$ & [160] \\
\hline Rats and Mice & Anthocyanins from bilberry provided moderate protection against Dox-induced cardiac and hematopoietic damage & [161] \\
\hline Mice & Blueberry extract showed antinociceptive activity & [162] \\
\hline Mice & Bilberry extract showed protective effective against endotoxin-induce uveitis & [163] \\
\hline Mice & Anthocyanins from lowbush blueberry showed hypoglycemic activity & [164] \\
\hline Mice & V. oxycoccos berries extract showed preventive effect on acetic acid-induced colitis & [167] \\
\hline Mice & $\begin{array}{l}\text { V. ashei berries extract showed improvement performance in memory tasks and had protective effects on brain } \\
\text { DNA }\end{array}$ & [168] \\
\hline Mice & extract of $V$. myrtillus significantly decrease the level of glucose and fructosamine in alloxan induced NOD & [169] \\
\hline Rats & $\begin{array}{l}\text { Anthocyanosides from } V \text {. myrtillus appear to be effective in preventing the increase in capillary filtration of } \\
\text { albumin (CFA) and the failure of lymphatic uptake of interstitial albumin }\end{array}$ & [170] \\
\hline Mice & Extract from $V$. ashei produced antinociceptive effects & [162] \\
\hline Mice & $\begin{array}{l}\text { Extract from } V \text {. corymbosum did not induce in vivo DNA damage in peripheral blood cells of } 12 \text {-day old female or } \\
\text { male Swiss mice but the micronucleus assay indicated that the extract presented clastogenic or aneugenic effects }\end{array}$ & [171] \\
\hline
\end{tabular}




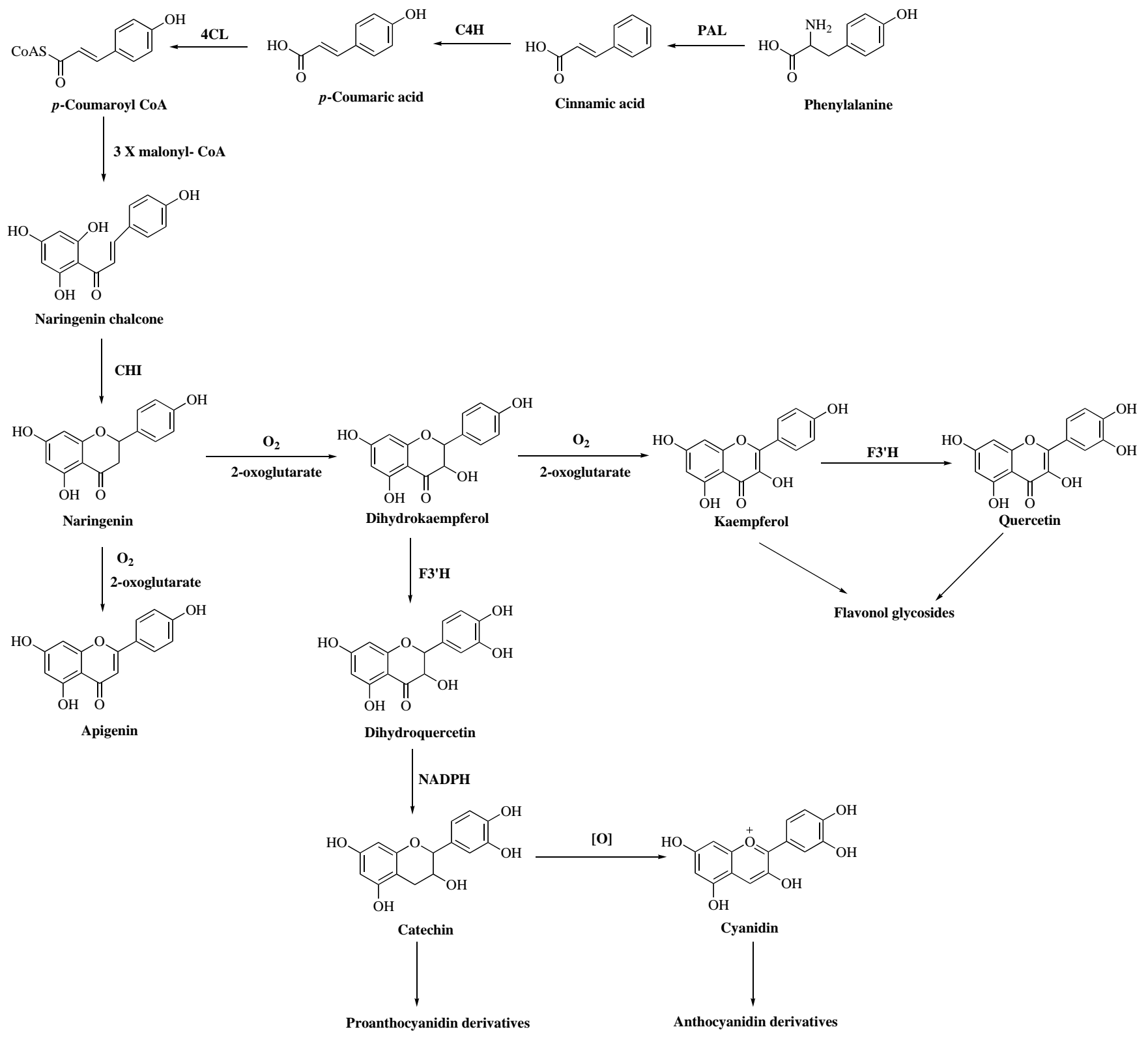

Fig. (3). Biosynthesis pathway of anthocyanidins and flavonoids.

or dihydroflavonol stage, and methylation, glycosylation, and dimethylallylation are also possible, increasing the range of compounds enormously.

\section{CONCLUSIONS}

The chemical constituents of the genus Vaccinium are best known for the production of anthocyanins and flavonoids which represent a class of important antioxidants, anti-inflammatory, antitumor, antiviral vasoprotective, and antifungal. A significant role of anthocyanins and flavonoids that have been under very active research recently, is their possible beneficial influence on human health. Anthocyanins and flavonoids have been found to own potent antioxidant and free radical scavenging activities in vitro. There is growing evidence from human consumption studies supporting a protective role of flavonoids in cardiovascular diseases and cancer. In recent years, many papers have been published on the in vitro antioxidant activity of anthocyanins and flavonoids and their other functions, as well as studies assessing the correlation between their antioxidant capacity and chemical structure. Some of the extract and individual compounds isolated from the genus Vaccinium have showed apparently biological activities. For example, the extract of bog whortleberry were excellent antioxidants (inhibition > $90 \%$ ) against the oxidation of human LDL at both concentration $(2.5$ and $7.5 \mu \mathrm{g} / \mathrm{mL})$ and the extract of fruit or leaf extracts of Vaccinium spp., were found to induce apoptosis in cancer cells and to inhibit human leukemia and breast, colon, lung, and prostate cancer cells in vitro. However, because of the wide variety of different flavonoids, their possible interactions with other substances, and the complexity of their metabolism in the human system, more research in this area is still needed. As the leaves and fruits of Vaccinium species are a rich source of phenolic compounds, they can in the future serve as a commercial 
source of specific compounds or fractions for pharmaceutics, cosmetics and natural product markets.

\section{ACKNOWLEDGEMENTS}

This work was funded by the USDA grant 2008-3892819308 and Stephen F. Austin State University.

\section{ABBREVIATIONS}

$\begin{array}{ll}\text { CFA } & =\text { Capillary filtration of albumin } \\ \text { CHI } & =\text { Chalcone isomerase } \\ \text { COX-2 } & =\text { Cyclooxygenase-2 } \\ \text { DPPH } & =2,2-\text { Diphenyl-1-picrylhydrazyl } \\ \text { HSCs } & =\text { Hematopoietic stem cells } \\ \text { I } \mathrm{BB} \alpha & =\text { Inhibitor Kappa B alpha } \\ \text { LDL } & =\text { Low density lipoproteins } \\ \text { NOD } & =\text { Non-obese diabetic } \\ \text { RBC } & =\text { Red blood cells } \\ \text { RRAC } & =\text { Oxygen radical absorbance capacity } \\ \text { TGF- } \beta 1 & =\text { Transforming growth factor-beta-1 } \\ \text { TNF- } \alpha & =\text { Tumor necrosis factor-alpha } \\ \text { TOSC } & =\text { Total oxidant scavenging capacity }\end{array}$

\section{REFERENCES}

[1] Fang, W.P.; Zhang, Z. R. Flora Reipublicae Popularis Sinicae, 57(3). Science Press: Beijing, 1983.

[2] Kloet, V.E. Manual of the flowering plants of Hawaii. 1990, Bishop Museum Special Publication, No. 83, 591-595.

[3] Nickavar, B.; Amin, G.; Salehi-Sormagi, M.H. Anatomical study on Vaccinium arctostaphylos L. Die. Pharmazie, 2003, 58, 274278.

[4] Yan, X.; Murphy, B.T.; Hammond, G.B.; Vinson, J.A.; Neto, C.C. Antioxidant activities and antitumor screening of extracts from cranberry fruit (Vaccinium macrocarpon). J. Agric. Food Chem., 2002, 50 (21), 5844-5849.

[5] Murphy, B.T.; Mackinnon, S.L.; Yan, X.; Hammond, G.B.; Vaisberg, A.J.; Neto, C.C. Identification of triterpene hydroxycinnamates with in vitro antitumor activity from whole cranberry fruit (Vaccinium macrocarpon). J. Agric. Food Chem., 2003, 51, 3541-3545.

[6] Katsube, N.; Iwashita, K.; Tsushida, T.; Yamaki, K.; Kobori, M. Induction of apoptosis in cancer cells by bilberry (Vaccinium myrtillus) and the anthocyanins. J. Agric. Food Chem., 2003, 51, 68-75.

[7] Neto, C.C.; Krueger, C.G.; Lamoureaux, T.L.; Kondo, M.; Vaisberg, A.J.; Hurta, R.A.R.; Curtis, S.; Matchett, M.D.; Yeung, H.; Sweeney, M.I.; Reed, J.D. MALDI-TOF MS characterization of proanthocyanidins from cranberry fruit (Vaccinium macrocarpon) that inhibit tumor cell growth and matrix metalloproteinase expression in vitro. J. Sci. Food Agric., 2005, 86, 18-25.

[8] Faria, A.; Pestana, D.; Teixeira, D.; de Freitas, V.; Mateus, N.; Calhau, C. Blueberry anthocyanins and pyruvic acid adducts: anticancer properties in breast cancer cell lines. Phytother. Res., 2010, 24, 1862-1869

[9] Zhao, C.; Giusti, M.M.; Malik, M.; Moyer, M.P.; Magnuson, B. A. Effects of commercial anthocyanin-rich extracts on colonic cancer and nontumorigenic colonic cell growth. J. Aric. Food Chem., 2004, 52, 6122-6128.

[10] Yi, W.; Fischer, J.; Krewer, G.; Akoh, C.C. Phenolic compounds from blueberries can inhibit colon cancer cell proliferation and induce apoptosis. J. Agric. Food Chem., 2005, 53, 7320-7329.
[11] Matchett, M.D.; MacKinnon, S.L.; Sweeney, M.I.; Gottschall-Pass, K.T.; Hurta, R.A.R. Blueberry flavonoids inhibit matrix metalloproteinase activity in DU145 human prostate cancer cells. Biochem. Cell. Biol., 2005, 83, 637-643.

[12] MacLean, M.A.; Matchett, M.D.; Amoroso, J.; Neto, C.; Hurta, R. Cranberry (Vaccinium macrocarpon) flavonoids inhibit matrix metalloproteinases (MMPs) in human prostate cancer cells. FASEB. J., 2007, 21, 791-795.

[13] Sellappan, S.; Akoh, C.C.; Krewer, G. Phenolic compounds and antioxidant capacity of Georgia-grown blueberries and blackberries. J. Agric. Food Chem., 2002, 50, 2432-2438.

[14] Prior, R.L.; Cao, G.H.; Martin, A.; Sofic, E.; McEwen, J.; O'Brien, C.; Lischner, N.; Ehlenfeldt, M.; Kalt, W.; Krewer, G.; Mainland, C. M. ntioxidant capacity as influenced by total phenolic and anthocyanin content, maturity, and variety of Vaccinium species. $J$. Agric. Food Chem., 1998, 46, 2686-2693.

[15] Skupień, K.; Oszmianski, J.; Kostrzewa-Nowak, D.; Tarasiuk, J. In vitro antileukaemic activity of extracts from berry plant leaves against sensitive and multidrug resistant HL60 cells. Cancer. Lett., 2006, 236, 282-291.

[16] Pourrat, H.; Guichard, J.P.; Pourrat, A.; Lamaison, J.L. Anthocyanins and flavones from Vaccinium corymbosum L. leaves. Plantes Medicinales et Phytotherapie, 1978, 12, 212-16.

[17] Lietti, A.; Cristoni, A.; Picci, M. Studies on Vaccinium myrtillus anthocyanosides. I. Vasoprotective and antiinflammatory activity. Arzneimittel-Forschung, 1976, 26, 829-32.

[18] Pourrat, H.; Pourrat, A. Anthocyanin glycosides of Vaccinium corymbosum. Plantes Medicinales et Phytotherapie, 1973, 7, 2479.

[19] Ballinger, W.E.; Maness, E.P.; Kushman, L. J. Anthocyanins in ripe fruit of the highbush blueberry, Vaccinium corymbosum, L. J. Am. Soc. Hortic. Sci., 1970, 95, 283-5.

[20] Hamamatsu, S.; Yabe, K.; Nawa, Y. Compositions of anthocyanin and other flavonoids in cultured cells of rabbiteye blueberry (Vaccinium ashei Reade cv. Tifblue). Food Sci. Technol. Res., 2004, 10, 239-246.

[21] Laaksonen, O.; Sandell, M.; Kallio, H. Chemical factors contributing to orosensory profiles of bilberry (Vaccinium myrtillus) fractions. Eur. Food Res. Technol., 2010, 231, 271-285.

[22] Hokkanen, J.; Mattila, S.; Jaakola, L.; Pirttila, A.M.; Tolonen, A. Identification of phenolic compounds from lingonberry (Vaccinium vitis-idaea L.), bilberry (Vaccinium myrtillus L.) and hybrid bilberry (Vaccinium intermedium Ruthe L.) leaves. J. Agric. Food Chem., 2009, 57, 9437-9447.

[23] Latti, A.K.; Jaakola, L.; Riihinen, K.R.; Kainulainen, P.S Anthocyanin and flavonol variation in bog bilberries (Vaccinium uliginosum L.) in Finlan. J. Agric. Food Chem., 2009, 58, 427-433.

[24] Cabrita, L.; Anderson, O. M. Anthocyanins in blue berries of Vaccinium padifolium. Phytochemistry, 1999, 52, 1693-1696.

[25] Lenascu, L.M.C.; Balcu, L.; Segneanu, A.E.; Cata, A.; Damian, D. Anthocyanins profile of Vaccinium myrtillus alcoholic extracts revealed by electrospray ionization/mass spectrometry. Ovidius Univ. Ann. Chem., 2009, 20, 76-79.

[26] Cabrita, L.; Froystein, N.A.; Andersen, O.M. Anthocyanin trisaccharides in blue berries of Vaccinium padifolium. Food Chem., 2000, 69, 33-36.

[27] Du, Q.; Jerz, G.; Winterhalter, P. Isolation of two anthocyanin sambubiosides from bilberry (Vaccinium myrtillus) by high-speed counter-current chromatography. J. Chromatogr. A, 2004, 1045, 59-63.

[28] Ochmian, I.; Oszmianski, J.; Skupien, K. Chemical composition, phenolics, and firmness of small black fruit. J. Appl. Bot. Food Qual., 2009, 83, 64-69.

[29] Francis, F.J.; Harborne, J.B.; Barker, W.G. Anthocyanins in the lowbush blueberry, Vaccinium angustifolium. J. Food Sci., 1966, 31, 583-587.

[30] Ballinger, W.E.; Maness, E.P.; Ballington, J.R. Anthocyanin and total flavonol content of Vaccinium stamineum L. fruit. Sci. Hort. (Amsterdam), 1981, 15, 173-178.

[31] Andersen, O.M. Chromatographic separation of anthocyanins in cowberry (lingonberry) Vaccinium vites-idaea L. J. Food Sci., 1985, 50, 1230-1232.

[32] Andersen, O.M. Anthocyanins in fruits of Vaccinium japonicum. Phytochemistry, 1987, 26, 1220-1221.

[33] Andersen, O.M. Anthocyanins in fruits of Vaccinium uliginosum L. (bog whortleberry). J. Food Sci., 1987, 52, 665-666, 680. 
[34] Ballington, J.R.; Ballinger, W.E.; Maness, E.P.; Luby, J.J. Anthocyanin, aglycon and aglycon-sugar content of the fruits of five species of Vaccinium section Myrtillus. Can. J. Pl. Sci., 1988, 68, 241-246.

[35] Ballington, J.R.; Ballinger, W.E.; Maness, E.P.; Luby, J.J.; Kirkman, W.B.; Ballinger, W.E.; Maness, E.P. Anthocyanin, aglycon, and aglycon-sugar content in the fruits of temperate North American species of four sections in Vaccinium. J. Amer. Soc. Hort. Sci., 1988, 113, 746-749.

[36] Andersen, O.M. Anthocyanins in fruits of Vaccinium oxycoccus L. (small cranberry). J. Food Chem., 1989, 54, 383-384, 387.

[37] Kader, F.; Rovel, B.; Girardin, M.; Metche, M. Fractionation and identification of the phenolic compounds of highbush blueberries (Vaccinium corymbosum L.). Food Chem., 1996, 55, 35-40.

[38] Madhavi, D.L.; Bomser, J.; Smith, M.A.L.; Singletary, K. Isolation of bioactive constituents from Vaccinium myrtillus (bilberry) fruits and cell cultures. Pl. Sci., 1998, 131, 95-103.

[39] Meng, F.L.; Su, X.T.; Li, Y.D. Extraction, separation and structure identification of cyanidin-3-galactoside in fruit of Vaccinium vitisidaea. J. Jilin. Agric. Univ., 2004, 26, 529-531.

[40] Yang, G.X.; Fan, H.L.; Zheng, Y.N.; Li, Y.D. Separation and identification of the flavonoids in the fruit of Vaccinium uliginosum L. blueberry. J. Jilin. Agric. Univ., 2005, 27, 643-644, 648.

[41] Li, Y.N.; Meng, F.L.; Zheng, Y.N. Study of anthocyanins in fruit of different Vaccinium genotypes. Acta. Hort., 2006, 715, 589-593.

[42] Ek, S.; Kartimo, H.; Mattila, S.; Tolonen, A. Characterization of phenolic compounds from lingonberry (Vaccinium vitis-idaea). J. Agric. Food Chem., 2006, 54, 9834-9842.

[43] Latti, A. K.; Riihinen, K.R.; Kainulainen, P.S. Analysis of anthocyanin variation in wild populations of bilberry (Vaccinium myrtillus L.) in Finland. J. Agric. Food Chem., 2008, 56, 190-196.

[44] Maurizio, B.; Jessica, S.; Alastair, C.; Stephens, J.; McGhie, T.; Peter, A. The anthocyanin composition of different Vaccinium, Ribes and Rubus genotypes. BioFactors, 2008, 34, 13-21.

[45] Suomalainen, H.; Keranen, A.J.A. Study of anthocyanins from Vaccinium myrtillus L. Nature, 1961, 191, 498-499.

[46] Spela, M.; Tomaz, P.; Lea, G.; Darinka, K.; Andreja, V.; Natasa, P.U.; Veronika, A. Phenolics in Slovenian Bilberries (Vaccinium myrtillus L.) and Blueberries (Vaccinium corymbosum L.). J. Agric. Food Chem., 2011, 59, 6998-7004.

[47] Garzon, G.A.; Narvaez., C.E.; Riedl, K.M.; Schwartz, S. J. Chemical composition, anthocyanins, non-anthocyanin phenolics and antioxidant activity of wild bilberry (Vaccinium meridionale Swartz) from Colombia. Food Chem., 2010, 122, 980-986.

[48] Laetti, A.K.; Riihinen, K.R.; Jaakola, L. Phenolic compounds in berries and flowers of a natural hybrid between bilberry and lingonberry (Vaccinium intermedium Ruthe). Phytochemistry, 2011, 72, 810-815.

[49] Stepanova, T.A.; Fedorev, S.N.; Stonik, V.A.; Mechikova, G. Ya.; Kuzmich, A.S.; Ponomarenko, L.P.; Kalinovsky, A.I. Cancerpreventive activities of secondary metabolites from leaves of the bilberry Vaccinium smallii A. Gray. Phytother. Res., 2010, 24, 1730-1732.

[50] Ice, C. H.; Wender, S.H. Quercetin and its glycosides in leaves of Vaccinium myrtillus. J. Am. Chem. Soc., 1953, 75, 50-52.

[51] Yasue, M.; Itaya, M.; Hanai, S.; Toda, M. Constituents of the leaves of Vaccinium bracteatum. Nagoya-shiritsu Daigaku Yakugakubu Kiyo, 1958, 6, 30-32.

[52] Yasue, M.; Itaya, M.; Oshima, H.; Funahashi, S. The constituents of the leaves of Vaccinium bracteatum. Yakugahu Zasshi., 1965, $85,553-556$

[53] Bohm, B.A.; Koupai-Abyazani, M.R. Flavonoids and condensed tannins from leaves of Hawaiian Vaccinium reticulatum and $V$. calycinum (Ericaceae). Pac. Sci., 1994, 48, 458-463.

[54] Joo, W.K.; Doh, S.H.; Lim, S.S.; Shin, K. H.; Woo, W. S. Phenolic compounds from the leaves of Vaccinium koreanum. Nat. Prod. Sci, 1999, 5, 60-63.

[55] Wang, X.J.; Feng, Y.L.; Yan, X.Y. Extraction, separation and structure identification of chemical constituents from stem and leaves of Vaccinium vitis-idaea. Zhong. Cao. Yao, 2002, 33, 595596.

[56] Wang, L.; Yan, H. Y. Extraction and purification of flavone from the leaves of Vaccinium bracteatum Thunb. Shipin Yu Fajiao Gongye, 2004, 30, 120-125.

[57] Koskimaeki, J.J.; Hokkanen, J.; Jaakola, L.; Suorsa, M.; Tolonen, A.; Mattila, S.; Pirttilae, A.M.; Hohtola, A. Flavonoid biosynthesis and degradation play a role in early defence responses of bilberry (Vaccinium myrtillus) against biotic stress. Eur. J. Plant. Pathol., 2009, 125, 629-640.

[58] Mzhavanadze, V.V. Kaempferol glycosides from the leaves of the Cancasian bilberry, Vaccinium arctostaphylos. Soobshch Akad Nauk Gruz SSR, 1971, 62, 445-447.

[59] Kaminska, J. Flavonoid compounds in the leaves of the cranberry (Vaccinium vitis-idaea). Pol. J. Pharmacol. Pharmacy., 1966, 18 , 267-273.

[60] Puski, G.; Francis, F.J. Flavonol glycosides in cranberries. J. Food Sci., 1967, 32, 527-530.

[61] Gerhardt, G.; Sinnwell, V.; Kraus, L. Isolation of quercetin 3glucuronide from the leaves of whortleberry and bog bilberry. Planta. Med., 1989, 55, 200-201.

[62] Friedrich, H.; Schoenert, J. Phytochemical investigation of leaves and fruits of Vaccinium myrtillus. Planta. Med., 1973, 24, 90-100.

[63] Matsuo, Y.; Fujita, Y.; Ohnishi, S.; Tanaka, T.; Hirabaru, H.; Kai, T.; Sakaida, H.; Nishizono, S.; Kouno, I. Chemical constituents of the leaves of rabbiteye blueberry (Vaccinium ashei) and characterisation of polymeric proanthocyanidins containing phenylpropanoid units and A-type linkages. Food Chem., 2010 121, 1073-1079.

[64] Zhang, L.; Li, B.G.; Fu, H.W.; Li, Z.L.; Tian, J. K. Flavonoid glycosides from leaves of Vaccinium bracteatum Thunb. Chin. Pharm. J., 2009, 44, 1773-1776.

[65] Vvedenskaya, I.O.; Vorsa, N. Flavonoid composition over fruit development and maturation in American cranberry, Vaccinium macrocarpon Ait. Plant. Sci., 2004, 167, 1043-1054.

[66] Cui, Z. H.; Yuan, C.S. Flavones of Vaccinium uliginosum fruits. Fitoterapia, 1992, 63, 283

[67] Pan, Y.F.; Qu, W.J.; Li, J.G.; Gu, Y.B. Qualitative and quantitative analysis of flavonoid aglycones from fruit residue of Vaccinium vitis-idaea L. by HPLC. Nat. Prod. Res. Develop., 2005, 17, 642$644,641$.

[68] Vasco, C.; Riihinen, K.; Ruales, J.; Kamal-Eldin, A. Chemical composition and phenolic compound profile of Mortino (Vaccinium floribundum Kunth). J. Agric. Food Chem., 2009, 57, 8274-8281.

[69] Vvedenskaya, I.O.; Rosen, R.T.; Guido, J.E.; Russell, D.J.; Mills, K. A.; Vorsa, N. Characterization of flavonols in cranberry (Vaccinium macrocarpon) powder. J. Agric. Food Chem., 2004, 52, 188-195.

[70] Lehtonen, H.M.; Lehtinen, O.; Suomela, J.P.; Viitanen, M.; Kallio, H. Flavonol glycosides of sea buckthorn (Hippophae rhamnoides ssp. sinensis) and lingonberry (Vaccinium vitis-idaea) are bioavailable in humans and monoglucuronidated for excretion. $J$. Agric. Food Chem., 2010, 58, 620-627.

[71] Denev, P.; Yanakieva, I.; Krachanova, M. Content of some polyphenol components in Bulgarian red fruits. Nauchni TrudoveUniversitet po Khranitelni Tekhnologii, 2009, 56, 456-462.

[72] Mechikova, G.Y.; Kuzmich, A.S.; Ponomarenko, L.P.; Kalinovsky, A.I.; Stepanova, T.A.; Fedorov, S.N.; Stonik, V.A. Cancerpreventive activities of secondary metabolites from leaves of the bilberry Vaccinium smallii A. Gray. Phytother. Res., 2010, 24, 1730-1732.

[73] Li, Z.L.; Zhang, L.; Tian, J. K.; Zhou, W.M. Studies on chemical constituents from leaves of Vaccinium bracteatum. L. J. Chin. Mat. Med., 2008, 33, 2087-2089.

[74] Wang, L.; Yao, H.Y.; Chen, Z.X. Extraction, separation, purification and characterization of flavonoid monomers from melanin of Vaccinium bracteatum. Patent, 2006, CN 1844116 A 20061011.

[75] Wang, L.; Yao, H.Y. Flavones in Vaccinium bracteatum Thunb. leaves. Nat. Prod. Res. Develop., 2007, 19, 989-990.

[76] Anna, S.; Laurence, V.N.; Max, H. Isolation and biological activities of lyoniside from rhizomes and stems of Vaccinium myrtillus. Phytochem. Lett., 2011, 4, 138-143

[77] Cesoniene, L.; Daubaras, R.; Jasutiene, I.; Vencloviene, J.; Miliauskiene, I. Evaluation of the biochemical components and chromatic properties of the juice of Vaccinium macrocarpon Aiton and Vaccinium oxycoccos L. Plant. Food Hum. Nutr., 2011, 66, 238-244.

[78] Su, M.S.; Chien, P.J. Aroma impact components of rabbiteye blueberry (Vaccinium ashei) vinegars. Food Chem., 2010, 119 923-928. 
[79] Chikako, M.; Kaori, Y.; Haruki, K.; Junei, K.; Toshihiro, N.; Masateru, O. Two novel antioxidant ortho-benzoyloxyphenyl acetic acid derivatives from the fruit of Vaccinium uliginosum. Food Sci. Technol. Res., 2007, 13, 215-20.

[80] Ono, M.; Masuoka, C.; Koto, M.; Tateishi, M.; Komatsu, H.; Kobayashi, H.; Igoshi, K.; Ito, Y.; Okawa, M.; Nohara, T. Antioxidant ortho- benzoyloxyphenyl acetic acid ester, vaccihein A, from the fruit of rabbiteye blueberry (Vaccinium ashei). Chem. Pharm. Bull., 2002, 50, 1416-1417.

[81] Turner, A.; Chen, S.N.; Nikolic, D.; van Breemen, R.; Farnsworth, N.R.; Pauli, G.F. Coumaroyl iridoids and a depside from Cranberry (Vaccinium macrocarpon). J. Nat. Prod., 2007, 70, 253-258.

[82] Jensen, H.D.; Krogfelt, K.A.; Cornett, C.; Hansen, S.H.; Christensen, S.B. Hydrophilic carboxylic acids and iridoid glycosides in the juice of American and European Cranberries (Vaccinium macrocarpon and $V$. oxycoccus), Lingonberries ( $V$. vitis-idaea), and Blueberries (V. myrtillus). J. Agric. Food Chem., 2002, 50, 6871-6874.

[83] Sakakibara, J.; Koto, T.; Yasue, M. Studies on the constituents of Vaccinium bracteatum Thunb. II. On the constituents of the flowers, particularly on the structure of vaccinoside, a new iridoid glycoside. Yakugaku Zasshi, 1973, 93, 164-70.

[84] Ono, M.; Kaiya, M.; Komatsu, H.; Igoshi, K.; Kobayashi, H.; Ito, Y.; Nohara, T. Cytotoxic triterpenes and sterol from the fruit of rabbiteye blueberry (Vaccinium ashei). Food Sci. Technol. Res., 2004, 10, 56-59.

[85] Piironen, V., Toivo, J.; Puupponen-Pimia, R.; Lampi, A. M. Plant sterols in vegetables, fruits and berries. J. Agric. Food Chem., 2003, 83, 330-337.

[86] Yang, B.; Koponen, J.; Tahvonen, R.; Kallio, H. Plant sterols in seeds of two species of Vaccinium (V. myrtillus and V. vitis-idaea) naturally distributed in Finland. Eur. Food Res. Technol., 2003, 216, 34-38.

[87] Koponen, J.; Kallio, H.; Yang, B.; Tahvonen, R. Plant sterols in finnish blueberry (Vaccinium myrtillus L.) and lingonberry (Vaccinium vitis-idaea L.) seed oils. Spec. Publ-Royal. Soc. Chem., 2001, 269, 233-236.

[88] Sheth, K.; Constantine, G. H., J.; Williams, D.K.; Catalfomo, P. Root triterpenes of Vaccinium species. Phytochemistry, 1968, 7, 1379-83.

[89] Murrhy, B.T.; Mackinnon, S.L.; Yan, X.J.; Hammond, G.B.; Vaisberg, A.J.; Neto, C.C. Identification of triterpene hydroxycinnamates with in vito antitumor activity from whole cranberry fruit (Vaccinium macrocarpon). J. Agric. Food Chem., 2003, 51, 3541-3545.

[90] Jaldappagari, S.; Motohashi, N.; Gangeenahalli, M.P.; Naismith, J. H. Bioactive mechanism of interaction between anthocyanins and macromolecules like DNA and proteins. Top. Heterocyc. Chem., 2008, $15,49-65$

[91] Wang, L.S., Stoner, G.D. Anthocyanins and their role in cancer prevention. Cancer. Lett., 2008, 269, 281-290.

[92] Moyer, R.A.; Hummer, K.E.; Finn, C.E.; Frei, B.; Wrolstad, R.E. Anthocyanins, phenolics, and antioxidant capacity in diverse small fruits: Vaccinium, Rubus, and Ribes. J. Agric. Food Chem., 2002, 50, 519-525.

[93] Prior, R.L.; Lazarus, S.A.; Cao, G; Muccitelli, H.; Hammerstone, J. F. Identification of procyanidins and anthocyanins in blueberries and cranberries (Vaccinium Spp.) using high-performance liquid chromatography/mass spectrometry. J. Agric. Food Chem., 2001, 49, 1270-1276.

[94] Christine, A.W.; Renee, J.G. Anthocyanins and other flavonoids. Nat. Prod. Rep., 2004, 21, 539-573.

[95] Scibisz, I.; Mitek, M. Antioxidant activity and phenolic compound content in dried highbush blueberries (Vaccinium corymbosum L.). Zywnosc., 2006, 13, 68-76./

[96] Lee, J.M.; Finn, C.E.; Wrolstad, R.E. Comparison of Anthocyanin Pigment and Other Phenolic Compounds of Vaccinium membranaceum and Vaccinium ovatum Native to the Pacific Northwest of North America. J. Agric. Food Chem., 2004, 52, 7039-7044.

[97] Nickavar, B.; Amin, G.; Salehi-Sormagi, M.H. Anthocyanins from Vaccinium arctostaphylos Berries. Pharm. Biol., 2004, 42, 289291.

[98] Suomalainen, H.; Keranen, A.J. A. The first anthocyanins appearing during the ripening of blueberries. Nature., 1961, 191, 498-499.
[99] Ienascu, I. M.C.; Balcu, I.; Segneaun, A.E.; Cata, A.; Damian, D. Anthocyanins profile of Vaccinium myrtillus alcoholic extracts revealed by electrospray ionization/mass spectrometry. Analele Universititatii "Ovidius" Constanta, Seria. Chimie., 2009, 20, 7679.

[100] Li, R.; Wang, P.; Guo, P.; Wang, Z.Y. Anthocyanin composition and content of the Vaccinium uliginosum berry. Food Chem., 2011, 125, 116-120.

[101] Latti, A.K.; Kainulainen, P.S.; Hayirlioglu-Ayaz, S.; Ayaz, F.A.; Riihinen, K.R. Characterization of anthocyanins in caucasian blueberries (Vaccinium arctostaphylos L.) native to Turkey. J. Agric. Food Chem., 2009, 57, 5244-5249.

[102] Witzell, J.; Gref, R; Nasholm, T. Plant-part specific and temporal variation in phenolic compounds of boreal bilberry (Vaccinium myrtillus) plants. Biochem. Syst. Ecol., 2003, 31, 115-127.

[103] Ochmian, I.; Oszmianski, J.; Skupien, K. Chemical composition, phenolics, and firmness of small black fruits. J. Appl. Bot. Food Qual., 2009, 83, 64-69.

[104] McIntyre, K. L.; Harris, C.S.; Saleem, A.; Beaulieu, L.P.; Ta, C.A.; Haddad, P.S.; Arnason, J.T. Seasonal phytochemical variation of anti-glycation principles in lowbush blueberry (Vaccinium angustifolium). Planta Med., 2009, 75, 286-292.

[105] Wang, S.Y.; Feng, R.T.; Bowman, L.; Lu, Y.Y.; Ballington, J.R. Ding, M. Antioxidant activity of Vaccinium stamineum: exhibition of anticancer capability in human lung and leukemia cells. Planta Med., 2007, 73, 451-460.

[106] Mzhavanadze, V.V. Flavonol glycosides of Caucasian blueberry (Vaccinium arctostaphylos) leaves. Biokhim. Rast., 1973, 1, 247 50 .

[107] Cesoniene, L.; Daubaras, R.; Jasutiene, I.; Vencloviene, J.; Miliauskiene, I. Evaluation of the biochemical components and chromatic properties of the juice of Vaccinium macrocarpon Aiton and Vaccinium oxycoccos L. Plant Food Hum. Nutr., 2011, 66 , 238-244.

[108] Shelyuto, V.L.; Glyzin, V.I.; Smirnova, L.P.; Kozyrev, I.A. Flavonoids of Oxycoccus quadripetalis. Chem. Nat. Compd., 1975, 11,515-16.

[109] Ranger, C.M.; Singh, A.P.; Johnson-Cicalese, J.; Polavarapu, S.; Vorsa, N. Intraspecific variation in aphid resistance and constitutive phenolics exhibited by the wild blueberry Vaccinium darrowi. J. Chem. Ecol., 2007, 33, 711-729.

[110] Wang, L.; Yao, H.Y.; Yao, H.Y.; Chen, Z.X. Isolation, purification and identification of flavonoids in Vaccinium bracteatum Thunb leaves. Chem. Ind. For. Prod., 2007, 27, 121-123.

[111] Latti, A.K.; Kainulainen, L.; Riihinen, K.R.; Kainulainen, P.S. Hayirlioglu-Ayaz, S.; Ayaz, F.A.; Riihinen, K.R. Characterization of anthocyanins in caucasian blueberries (Vaccinium arctostaphylos L.) native to turkey. J. Agric. Food Chem., 2009, 57, 5244-5249.

[112] Gu, L.; Kelm, M.A.; Hammerstone, J.F.; Beecher, G.; Holden, J.; Haytowitz, D.; Prior, R.L. Screening of foods containing proanthocyanidins and their structural characterization using LCMS/MS and thiolytic degradation. J. Agric. Food Chem., 2003, 51, 7513-7521.

[113] Gu, L.; Kelm, M.A.; Hammerstone, J.F.; Beecher, G.; Holden, J. Haytowitz, D.; Gebhardt, S.; Prior, R.L. Concentrations of proanthocyanidins in common foods and estimations of normal consumption. J. Nutr., 2004, 134, 613-617.

[114] Schmidt, B.M.; Howell, A.B.; McEniry, B.; Knight, C.T.; Seigler D.; Erdman, J.W.J.; Lila, M.A. Effective separation of potent antiproliferation and antiadhesion components from wild blueberry (Vaccinium angustifolium Ait.) fruits. J. Agric. Food Chem., 2004, 52, 6433-6442.

[115] Prior, R.L.; Lazarus, S.A.; Cao, G.; Muccitelli, H.; Hammerstone, J. F. Identification of procyanidins and anthocyanins in blueberries and cranberries (Vaccinium spp.) using highperformance liquid chromatography/mass spectrometry. J. Agric. Food Chem., 2001, 49, 1270-1276.

[116] Foo, L.Y.; Lu, Y.; Howell, A.B.; Vorsa, N. A-type proanthocyanidin trimers from cranberry that inhibit adherence of uropathogenic P-fimbriated Escherichia coli. J. Nat. Prod., 2000, 63, 1225-1228.

[117] Morimoto, S.; Nonaka, G.I.; Nishioka, I. Tannins and related compounds. LX. Isolation and characterization of proanthocyanidins with a double-linked unit from Vaccinium Vitisidaea L. Chem. Pharm. Bull., 1988, 36, 33-38. 
[118] Maatta-Riihinen, K.R.; Kähkönen, M.R.; Torronen, A.R.; Heinonen, I.M. Catechins and Procyanidins in Berries of Vaccinium Species and Their Antioxidant Activity. J. Agric. Food Chem., 2005, 53, 8485-8491.

[119] Juho, H.; Sampo, M.; Laura, J.; Anna, M.P.; Ari, T. Identification of phenolic compounds from lingonberry (Vaccinium vitis-idaea L.), bilberry (Vaccinium myrtillus L.) and hybrid bilberry (Vaccinium $\times$ intermedium Ruthe L.) leaves. J. Agric. Food Chem., 2009, 57, 9437-9447.

[120] Wang, H.; Nair, M.G.; Strasburg, G.M.; Chang, Y.C.; Booren, A.M.; Gray, J.I.; DeWitt, D.L. Antioxidant and antiinflammatory activities of anthocyanins and their aglycon, cyanidin, from Tart Cherries. J. Nat. Prod., 1999, 62, 294-296.

[121] Jepson, R.G.; Craig, J.C. A systematic review of the evidence for cranberries and blueberries in UTI prevention. Mol. Nutr. Food Res., 2007, 51, 738-745.

[122] Yuan, W.; Zhou, L.J.; Deng, G.R.; Wang, P.; Creech, D.; Li, S.Y. Anthocyanidins, phenolics, and antioxidant capacity of Vaccnium L. in Texa, USA. Pharm. C., 2011, 2, 11-13.

[123] Arts, I.C.; Hollman, P. Polyphenols and disease risk in epidemiologic studies. Am. J. Clin. Nutr., 2005, 81, 317-325.

[124] Mertens, A.; Holvoet, P. Oxidized LDL and HDL: Antagonists in atherothrombosis. FASEB J., 2001, 15, 2073-2084.

[125] Reed, J. Cranberry flavonoids, atherosclerosis and cardiovascular health. Crit. Rev. Food Sci. Nutr., 2002, 42, 301-316.

[126] Heinonen, M. Antioxidant activity and antimicrobial effect of berry phenolics - a Finnish perspective. Mol. Nutr. Food Res., 2007, 51, 684-91.

[127] Wang, S.Y.; Jiao, H. Scavenging capacity of berry crops on superoxide radicals, hydrogen peroxide, hydroxyl radicals, and singlet oxygen. J. Agr. Food Chem., 2000, 48, 5677-84

[128] Wu, X.; Prior, R.L. Systematic identification and characterization of anthocyanins by HPLC-ESI-MS-MS in common foods in the US - Fruits and berries. J. Agr. Food Chem., 2005, 53, 2589-2599.

[129] Seeram, N.P.; Heber, D. Impact of berry phytochemicals on human health: Effects beyond antioxidant. ACS Sym. Ser., 2007, 956, 326336.

[130] Singh, B.; Bhat, T.K.; Singh, B. Potential therapeutic applications of some antinutritional plant secondary metabolites. J. Agric. Food Chem., 2003, 51, 5579-5597.

[131] Pappasa, E.; Schaicha, K.M. Phytochemicals of cranberries and cranberry products: characterization, potential health effects, and processing stability. Crit. Rev. Food Sci., 2009, 49, 741-781.

[132] Cote, J.; Caillet, S.; Doyon, G.; Sylvain, J.F.; Lacroix, M. Bioactive compounds in cranberries and their biological properties. Crit. Rev. Food Sci., 2010, 50, 666-679.

[133] Tao, X.Y.; Lu, Y.H.; Zhou, W.Y. Protective effects of Vaccinium myrtillus L. and Vaccinium uliginosum L. on oxidative damage in neural cells. Chin. J. Clin. Rehab., 2005, 9, 50-52.

[134] Yao, Y.; Vieira, A. Protective activities of Vaccinium antioxidants with potential relevance to mitochondrial dysfunction and neurotoxicity. Neurotoxicology, 2007, 28, 93-100.

[135] Dai, J.; Mumper, R.J. Plant phenolics: extraction, analysis and their antioxidant and anticancer properties. Molecules 2010, 15, 73137352 .

[136] Bomser, J.; Madhavi, D.L.; Singletary, K.; Smith, M.A. In vitro anticancer activity of fruit extracts from Vaccinium species. Planta Med., 1996, 62, 212-216.

[137] Seeram, N.P.; Adams, L.S.; Zhang, Y.; Lee, R.; Sand, D.; Scheuller, H.S.; Heber, D. Blackberry, black raspberry, blueberry, cranberry, red raspberry, and strawberry extracts inhibit growth and stimulate apoptosis of human cancer cells in vitro. J. Agric. Food Chem., 2006, 54, 9329-39.

[138] Yi, W.; Akoh, C.C.; Fischer, J.; Krewer, G. Absorption of anthocyanins from blueberry extracts by caco-2 human intestinal cell monolayers. J. Agric. Food Chem., 2006, 54, 5651-8.

[139] Matchett, M.D.; MacKinnon, S.L.; Sweeney, M.I.; Gottschall-Pass, K.T.; Hurta, R.A. Blueberry flavonoids inhibit matrix metalloproteinase activity in DU145 human prostate cancer cells. Biochem. Cell Biol., 2005, 83, 637-43.

[140] Zu, X.Y.; Zhang, Z.Y.; Zhang, X.W.; Yoshioka, M.; Yang, Y.N.; $\mathrm{Li}, \mathrm{J}$. Anthocyanins extracted from Chinese blueberry (Vaccinium uliginosum L.) and its anticancer effects on DLD-1 and COLO205 cells. Chin. Med. J. (Engl), 2010, 123, 2714-2719.
[141] Narayansingh, R.; Hurta, R.A.R. Cranberry extract and quercetin modulate the expression of cyclooxygenase-2 (COX-2) and $\mathrm{I} \kappa \mathrm{B} \alpha$ in human colon cancer cells. J. Sci. Food Agr., 2009, 89, 542-547.

[142] Feng, R.; Bowman, L.L.; Lu, Y.; Leonard, S.S.; Shi, X.; Jiang, B. H.; Castranova, V.; Vallyathan, V.; Ding, M. Blackberry extracts inhibit activating protein 1 activation and cell transformation by perturbing the mitogenic signaling pathway. Nutr. Cancer, 2004, 50, 80-9.

[143] Cote, J.; Caillet, S.; Doyon, G.; Dussault, D.; Sylvain, J.-F.; Lacroix, M. Antimicrobial effect of cranberry juice and extracts. Food Control, 2011, 22, 1413-1418.

[144] Lacombe, A.; Wu, V.C.H.; Tyler, S.; Edwards, K. Antimicrobial action of the American cranberry constituents; phenolics, anthocyanins, and organic acids, against Escherichia coli O157:H7. Int. J. Food Microbiol., 2010, 139, 102-107.

[145] Viskelis, P.; Rubinskiene, M.; Jasutiene, I.; Sarkinas, A.; Daubaras, R.; Cesoniene, L. Anthocyanins, antioxidative, and antimicrobial properties of American cranberry (Vaccinium macrocarpon Ait.) and their press cakes. J. Food Sci., 2009, 74, 157-161.

[146] Ho, K.Y.; Tsai, C.C.; Huang, J.S.; Chen, C.P.; Lin, T.C.; Lin, C.C. Antimicrobial activity of tannin components from Vaccinium vitisidaea. J. Pharm. Pharmacol., 2001, 53, 187-191.

[147] Bodet, C.; Grenier, D.; Chandad, F.; Ofek, I.; Steinberg, D.; Weiss, E. I. Potential oral health benefits of cranberry. Crit. Rev. Food Sci. 2008, 48, 672-680.

[148] Beighton, D. The complex oral microflora of high-risk individuals and groups and its role in the caries process. Community Dent. Oral, 2005, 33, 248-255.

[149] Zafriri, D.; Ofek, I.; Adar, R.; Pocino, M.; Sharon, N. Inhibitory activity of cranberry juice on adherence of type 1 and type $\mathrm{P}$ fimbriated Escherichia coli to eucaryotic cells. Antimicrob. Agents Ch. 1989, 33, 92-98.

[150] Sharon, N.; Ofek, I. Fighting infectious diseases with inhibitors of microbial adhesion to host tissues. Crit. Rev. Food Sci. 2002, 42, 267-72.

[151] Lipson, S.M.; Sethi, L.; Cohen, P.; Gordon, R.E.; Tan, I.P.; Burdowski, A.; Stotzky, G. Antiviral effects on bacteriophages and rotavirus by cranberry juice. Phytomedicine, 2007, 14, 23-30.

[152] Qadana, F.; Verspohl, E.J.; Nahrstedt, A.; Petereit, F.; Matalka, K. Z. Cinchonain Ib isolated from Eriobotrya japonica induces insulin secretion in vitro and in vivo. J. Ethnopharmacol., 2009, 124, 224227.

[153] Martineau, L.C.; Couture, A.; Spoor, D.; Benhaddou-Andaloussi, A.; Harris, C.; Meddah, B.; Leduc, C.; Burt, A.; Vuong, T.; Mai Le, P.; Prentki, M.; Bennett, S.A.; Arnason, J.T.; Haddad, P.S Antidiabetic properties of the Canadian lowbush blueberry Vaccinium angustifolium Ait. Phytomedicine, 2006, 13, 612-623.

[154] Torri, E.; Lemos, M.; Caliari, V.; Kassuya, C. A. L.; Bastos, J Andrade, S. F. Anti-inflammatory and antinociceptive properties of blueberry extract (Vaccinium corymbosum). J. Pharm. Pharmacol., 2007, 59, 591-596.

[155] Kylli, P.; Nohynek, L.; Puupponen-Pimia, R.; Westerlund-Wikstro, B.; Tiinanen, L.; Welling, J.; Moilanen, E.; Heinonen, M. Lingonberry (Vaccinium vitis-idaea) and European Cranberry (Vaccinium microcarpon) proanthocyanidins: isolation, identification, and bioactivities. J. Agric. Food Chem., 2011, 59, 3373-3384.

[156] Ming, D.S.; Lopez, A.; Hillhouse, B.J.; French, C.J.; Hudson, J.B.; Towers, G.H.N. Bioactive constituents from Iryanthera megistophylla. J. Nat. Prod., 2002, 65, 1412-1416.

[157] Bell, D.R.; Gochenaur, K. Direct vasoactive and vasoprotective properties of anthocyanin-rich extracts. J. Appl. Physiol., 2006, 100, 1164-1170.

[158] Katsunori, Y.; Maki, S.; Koichi, U. Anthocyanins from bilberry (Vaccinium myrtillus L.) alleviate pruritus in a mouse model of chronic allergic contact dermatitiss. Pharm. Res., 2011, 3, 173177.

[159] Kenjirou, O.; Atsushi, O.; Junji, T.; Saori, K.; Hideaki, H. The protective effect and action mechanism of Vaccinium myrtillus $\mathrm{L}$. on Gastric Ulcer in mice. Phytother. Res., 2011, 25, 1160-1165.

[160] Robert, D.; Hrvoje, J. Effects of standardized bilberry fruit extract (Mirtoselect) on resolution of $\mathrm{CCl} 4$-induced liver fibrosis in mice. Food Chem. Toxicol., 2011, 49, 848-854.

[161] Choi, E.H.; Park, J.H.; Kim, M.K.; Chun, H.S. Alleviation of doxorubicin-induced toxicities by anthocyanin-rich bilberry 
(Vaccinium myrtillus L.) extract in rats and mice. Biofactors, 2010, 36, 319-27.

[162] Ramirez, M.R.; Guterres, L.; Dickel, O.E.; De Castro, M.R.; Henriques, A.T.; De Souza, M.M.; Barros, D.M. Preliminary studies on the antinociceptive activity of Vaccinium ashei berry in experimental animal models. J. Med. Food, 2010, 13, 336-342.

[163] Yao, N.; Lan, F.; He, R.R.; Kurihara, H. Protective effects of bilberry (Vaccinium myrtillus L.) extract against endotoxin-induced uveitis in mice. J. Agric. Food Chem., 2010, 58, 4731-4736.

[164] Grace, M.H.; Ribnicky, D.M.; Kuhn, P.; Poulev, A.; Logendra, S.; Yousef, G.; Raskin, G.; Lila, M. A. Hypoglycemic activity of a novel anthocyanin-rich formulation from lowbush blueberry, Vaccinium angustifolium Aiton. Phytomedicine, 2009, 16, 406-415.

[165] Bao, L.; Yao, X.S.; Yau, C.C.; Tsi, D.; Chia, C.S.; Nagai, H.; Kurihara, H. Protective effects of bilberry (Vaccinium myrtillus L.) extract on restraint stress-induced liver damage in mice. J. Agric. Food Chem., 2008, 56, 7803-7807.

[166] Bao, L.; Yao, X.S.; Tsi, D.; Yau, C.C.; Chia, C.S.; Nagai, H.; Kurihara, H. Protective effects of bilberry (Vaccinium myrtillus L.) extract on $\mathrm{KBrO} 3$-induced kidney damage in mice. J. Agric. Food Chem., 2008, 56, 420-425.

[167] Popov, S.V.; Markov, P.A.; Nikitina, I.R.; Petrishev, S.; Smirnov, V.; Ovodov, Y.S. Preventive effect of a pectic polysaccharide of the common cranberry Vaccinium oxycoccos L. on acetic acid- induced colitis in mice. World J. Gastroentero., 2006, 12, 66466651.

[168] Barros, D.; Amaral, O.B.; Izquierdo, I.; Geracitano, L.; Raseira, M.; Henriques, A.T.; Ramirez, M.R. Behavioral and genoprotective effects of Vaccinium berries intake in mice. Pharmacol., Biochem. Behav., 2006, 84, 229-234

[169] Petlevski, R.; Hadija, M.; Slijepevi, M. Effect of'antidia2 betis'herbalp reparation on serum glucose and fructosamine in NOD mice. J. Ethnopharmacol., 2001, 75, 181-184.

[170] Francine, C.B.; Paul, E.V.; Hatem, B.; Roger, L.; Jean-Francois, D. L.; Claudine, H. L.; Abraham, B. In vivo sequential study of skeleral muscle capillary permeability in diabetic rats: effect of anthocyanosides. Metabolism, 2000, 49, 880-885.

[171] Freitas, P.S.; de Andrade, S.F.; Maistro, E.L. Evaluation of the in vivo mutagenic potential of hydroalcoholic extracts of the northern highbush blueberry (Vaccinium corymbosum L. Ericales, Ericaceae) on peripheral blood cells of Swiss mice (Mus musculus Rodentia, Muridae). Genet. Mole. Biol., 2008, 31, 555-560.

[172] Hoffmann, L.; Besseau, S.; Geoffroy, P.; Ritzenthaler, C.; Meyer, D.; Lapierre, C.; Pollet, B.; Legrand, M. Silencing of hydroxycinnamoyl-coenzyme A shikimate/quinate hydroxycinnamoyl transferase affects phenylpropanoid biosynthesis. Plant Cell, 2004, 16, 1446-1465.

[173] Paul, M.D. Medicinal Natural Products. John Wiley \& Sons Ltd: NY, 2002, 149-150.

Received: October 26, 2011

Revised: November 24, 2011

Accepted: November 24, 2011

(c) Zushang Su. Licensee Bentham Open.

This is an open access article licensed under the terms of the Creative Commons Attribution Non-Commercial License (http: //creativecommons.org/licenses/bync/3.0/.) which permits unrestricted, non-commercial use, distribution and reproduction in any medium, provided the work is properly cited. 